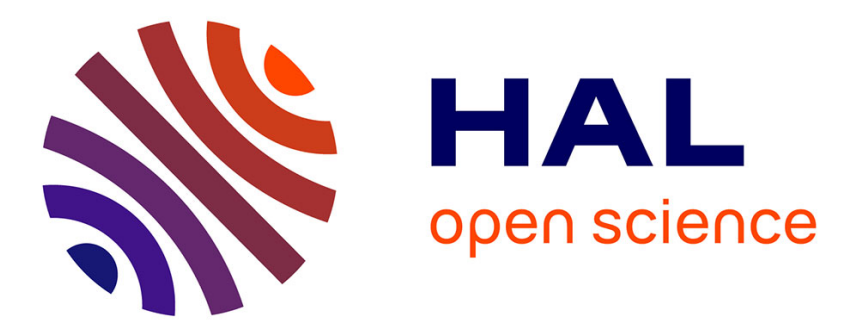

\title{
Progressive damage of a unidirectional composite with a viscoelastic matrix, observations and modelling
}

Natalia Kotelnikova-Weiler, Olivier Baverel, Nicolas Ducoulombier, Jean-François Caron

\section{- To cite this version:}

Natalia Kotelnikova-Weiler, Olivier Baverel, Nicolas Ducoulombier, Jean-François Caron. Progressive damage of a unidirectional composite with a viscoelastic matrix, observations and modelling. Composite Structures, 2018, 10.1016/j.compstruct.2017.12.067 . hal-02131852

\section{HAL Id: hal-02131852 \\ https://hal-enpc.archives-ouvertes.fr/hal-02131852}

Submitted on 16 May 2019

HAL is a multi-disciplinary open access archive for the deposit and dissemination of scientific research documents, whether they are published or not. The documents may come from teaching and research institutions in France or abroad, or from public or private research centers.
L'archive ouverte pluridisciplinaire HAL, est destinée au dépôt et à la diffusion de documents scientifiques de niveau recherche, publiés ou non, émanant des établissements d'enseignement et de recherche français ou étrangers, des laboratoires publics ou privés. 


\title{
Progressive damage of a unidirectional composite with a viscoelastic matrix, observations and modelling
}

\author{
Natalia Kotelnikova-Weiler, Olivier Baverel, Nicolas Ducoulombier, Jean-François Caron* \\ Université Paris-Est, Laboratoire Navier (UMR 8205), CNRS, ENPC, IFSTTAR, 6-8 av. Blaise Pascal, Cité Descartes, \\ Champs-sur-Marne, 77455 Marne-la-Vallée, France
}

\begin{abstract}
In this paper phenomenological observations of the creep rupture under maintained combined traction and torsion loading are first presented. They show the importance of the matrix's behavior in the long-term durability of the material. Understanding and foreseeing creep rupture of unidirectional fiber reinforced polymers (UD FRP) involves comprehension at the fibers' scale of various time-dependent interactions among the fibers and between the matrix and the fibers. Shear-lag models have been successfully applied in the modeling at the micro-scale of these interactions, some of them even introducing time dependence. Longterm durability of macro-scale structure demand further developments of such models to be able to predict the macro-damage cluster and their evolution. The aim of the present contribution that is an extension from existing models is to investigate the progressive damage of a 0o UD composite material subjected to combined shear-traction loading including a high number of interacting fibers, with a viscoelastic matrix, debondings and random distribution of fiber flaws. Results of simulations including different loadings and matrix viscoelastic properties will be shown and discussed for a better comprehension of the role of composite's components in the creep rupture phenomenon. In particular, the long-term influence of matrix's shear stiffness on the material's lifespan is shown, and the impact of an additional uniform shear stress is studied. This combined shear-traction loading is of interest in real-scale structures where shear stress can result from torsion or shear forces (such as those due to anchor points, misalignment and coupling). Moreover, this model is a first step to approach the long term failure of 0o composites subjected to torsion-bending loading, what is shown decisive in the second section of this work. Further experimental works in combined traction-torsion loadings are needed to validate this simulations with a specific attention on the identification of required parameters.
\end{abstract}

Keywords: Shear-lag model, stochastic fiber strength, viscoelastic matrix, debonding, stress transfer mechanism, damage cluster, stress redistribution, matrix shear stiffness, combined shear-traction loading, frictional shear stress, debonding criterion 


\section{Introduction}

Advantages of composite materials need no more presentation. Their ability to combine light weight, anisotropy and high performances are well known and their scope of applications is currently covering a wide range of fields from highly specialized aeronautical to widely spread civil engineering and construction. In load bearing functions composite materials are however combined with more traditional materials. Although long-term durability data and feedback on field applications of composite materials combined with other materials become available nowadays $[1,2]$, the application and development of composite materials as main load-bearing material is still strongly dependent on the durability studies and construction of valid models capable of predicting with a sufficiently high level of confidence the lifespan of a given composite, that is a given combination of a matrix, fibers and fiber-matrix interface treatment, subjected to a loading.

In the civil engineering and construction field, pultruded unidirectional composites are widely used and still offer a great potential. Construction manuals and calculation codes (EUROCOMP for example) prescribe 30\% maximum loading to guarantee security and safety in long-term applications where permanent loading or aggressive environments are applied (Karbhari et al. [3], 2003). Where greater loads are to be supported, composites are used in combination with other materials such as steel, concrete or wood [4-6]. All-composite structures have also been developed exploiting their durability and mechanical characteristics such as resistance to corrosion and humidity and high stiffness-to-weight or strength-to-weight ratios. For example $\mathrm{Wu}$ and his colleagues presented in their article (Wu et al. [7]) a bridge deck system made of pultruded tubular FRP profiles.

To make the most of the composite materials' characteristics, new structures needed to be developed. They make use of the anisotropy and combine high strength and flexibility. They are not a mere transposition of steel structures typologies to composite applications but are specifically designed for them. Bows, snowboards and skis in sport applications, pop-up tents (for example Quechua ${ }^{\circledR}$ tents are experimentally investigated in this paper) and hybrid beams (Hillman [8]) are good examples of such structures already developed and commercialized. Other applications are currently being studied: gridshell structures for light covering and building envelopes (Douthe et al. [9], Douthe et al. [10]).

One of the issues in the study of long-term behavior of composite materials is the almost infinite number of combinations between matrices and fibers leading to sometimes dramatically different performances. On the other hand, very low probabilities of failure are expected for engineering applications (up to $10^{-9}$ ) making experimental studies extremely expensive. Simulation and modeling are therefore essential in the forecast of a new material's lifespan. To make reliable models, the precise role of each component must be understood and validation procedures revealing this role must be defined. In composite materials' applications the

\footnotetext{
*Corresponding author

Email address: caron@enpc.fr (Jean-François Caron)
} 
load bearing elements are fibers but matrix's behavior can lead to completely different rupture mechanisms.

Influence of different material parameters on creep and creep rupture need to be clearly identified. Models' sensitivity to these parameters also need to be adjusted accordingly. In the first following part, original experiments which have motivated this numerical developments are shown. In a second part a review of existing modeling is proposed, leading to the presentation of the used model in a third part. It is an adaptation of different approaches that we have combined and extended with a debonding including timedependent friction. Finally, results of stochastic simulations including a high number of fibers, different loadings and matrix viscoelastic properties will be shown and discussed. In particular, the long-term influence of matrix's shear stiffness on the material's lifespan is shown, and the impact of an additional uniform shear stress is studied. This combined shear-traction loading is of interest in real-scale structures where shear stress can result from torsion or shear forces (such as those due to anchor points, misalignment and coupling).

\section{Experimental study and phenomenological observations}

Creep rupture is closely related to the viscoelastic properties of the matrix. In order to better understand the role of the matrix and the influence of its properties on the creep rupture, a series of experiments that highlight matrix's role has been lead (for further details see Kotelnikova-Weiler [11]). This was accomplished through direct loading of the resin via torsion on glass fiber reinforced vinylester composite unidirectional cylindrical 0 degree rods used in the pop-up tents Quechua ${ }^{\circledR}$. Indeed, torsion results in shear stresses acting on the resin. Static rupture in pure and combined torsion, compression and traction were first studied. These tests revealed three different rupture modes (figure 1). Material Data are given in table 1. Resin properties was given by the constructor and composites properties was tested on four samples.

\begin{tabular}{|c|c|c|c|c|}
\hline Material & Derakane 470 & Derakane 441 & GF + Derakane 470 & GF + Derakane 441 \\
\hline Tensile strength & $85 \mathrm{Mpa}$ & $90 \mathrm{MPa}$ & $846.4 \pm 27 \mathrm{MPa}$ & $602.13 \pm 24 \mathrm{MPa}$ \\
\hline Tensile Elongation & $3-4 \%$ & $5-6 \%$ & $2.311 \pm 0.06 \%$ & $1.616 \pm 0.07 \%$ \\
\hline Tensile Modulus & $3.6 \mathrm{GPa}$ & $3.3 \mathrm{GPa}$ & $36.1 \pm 0.04 \mathrm{GPa}$ & $37.7 \pm 0.09 \mathrm{GPa}$ \\
\hline
\end{tabular}

Table 1: Material Data of both vinylester resins and both composite rods

In pure compression the classic kink-band mode was observed. The sample stayed whole, the two edges of it remaining linked by the buckled band (figure 1 (a)). When combined torsion and compression were applied, at the rupture point, the sample crushed quasi-instantaneously leaving an almost clean fracture surface (figure $1(\mathrm{~b})$ ). In torsion-traction, the material is subjected to longitudinal cracking (figure 1 (c)) in the manner of pure torsion rupture. The rupture mode resulting from combined compression-torsion loading 


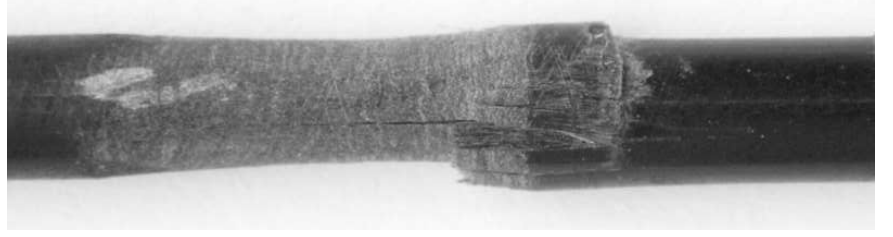

(a) Compression

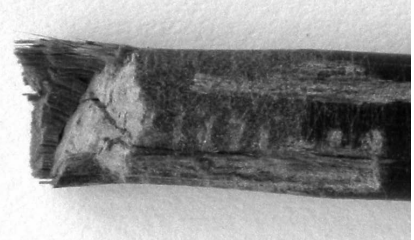

(b) Combined torsion-compression

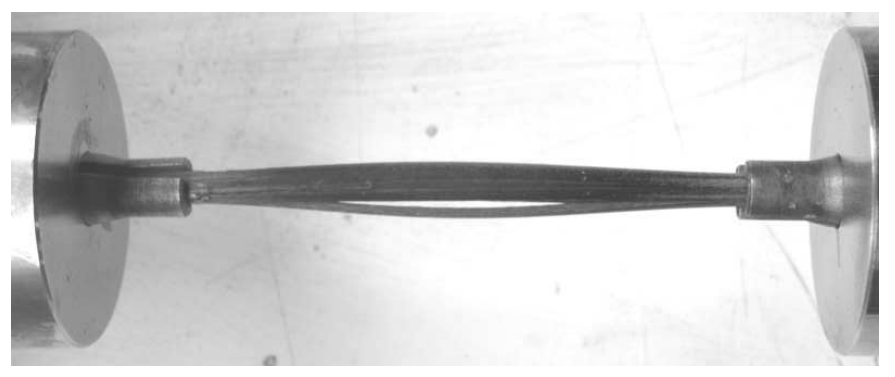

(c) Combined torsion-traction

Figure 1: Static rupture modes for different pure and combined loadings.

kept our attention. In this mode rupture is highly localized in a given material's section. Next long-term testing was performed to see if this localized rupture mode could occur in time.

For long-term testing a special device was designed. Pultruded cylindrical rod unidirectional 0 degree composite samples of $5 \mathrm{~mm}$ of diameter, used for the structure of the pop-up tent Quechua ${ }^{\circledR}$ were provided by the constructor, for testing under sustained combined torsion and bending (loaded samples have the shape of Euler's elastica: figure 2 (a)). Two different vinylester matrix systems were used. The stress state combines torsion-compression and torsion-traction. On the figure 2 (a), the point A allows free rotation around the axis perpendicular to the picture plane and the point B represents the location of the maximum loading. Loading levels in bending range between $40 \%$ and $70 \%$ of initial static strength and to each bending load level several different torsion loading levels were combined. Thus pairs of bending/torsion loadings are obtained. Torsion loading levels range between $31 \%$ and $110 \%$ of initial torsion elastic limit. Tests were carried out at ambient and elevated temperatures $\left(60^{\circ} \mathrm{C}\right)$. Rupture occurs after a certain period of time. The fracture surface is presented in the figure (2 (b)). This rupture mode can be compared to the pure bending creep rupture mode given in the figure $(2(\mathrm{c}))$. The pure bending creep rupture resembles the well-known 
72 brush-like static bending rupture mode of UD composite materials. Superimposing torsion to the bending

73 loading changes the rupture mode from the progressive brush-like (fig. 2 (c)) to the abrupt mode (fig. 2 (b)).

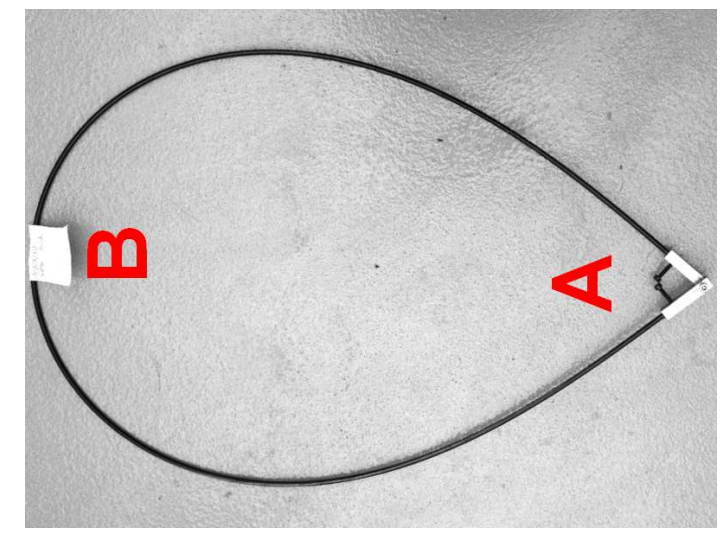

(a) Torsion-bending testing device

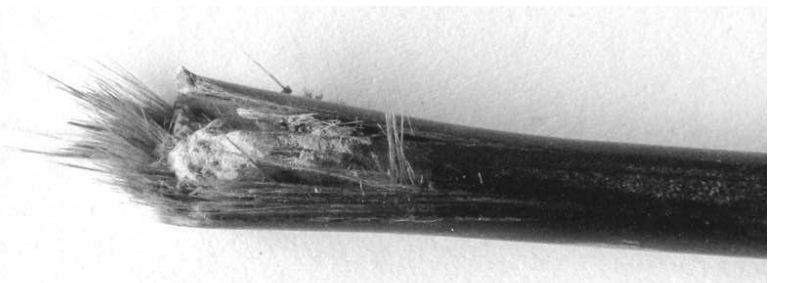

(b) Creep rupture in torsion-bending

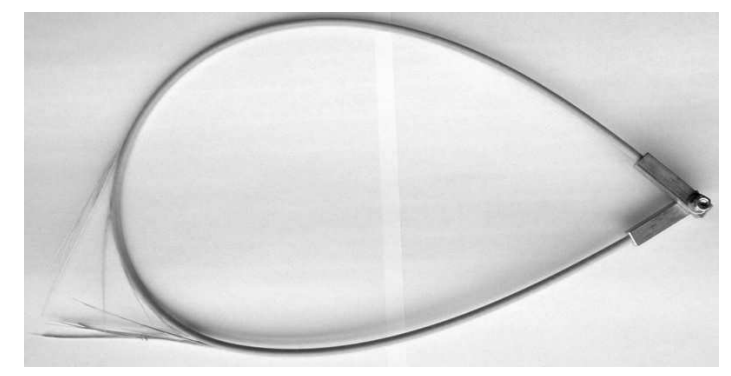

(c) Creep rupture in pure bending

Figure 2: Long-term testing in combined torsion-bending.

74

Main results of this experimental study will be summarized in this section.

- On the whole, results show that for a given testing temperature, the lifespan decreases with increasing torsion loading.

- It can also be clearly seen that torsion loading has a greater influence on the lifespan than bending loading. For example the lifespan of samples tested at $60^{\circ} \mathrm{C}$ at $50 \%$ bending loading and $62 \%$ torsion 
loading is greater than 17000 hours whereas samples tested at $50 \%$ bending and $77 \%$ torsion loading have a mean lifespan lower than 1 hour.

- Elevated temperature also diminishes the lifespan of samples tested in the same loading conditions. For example samples tested at $50 \%$ bending and $77 \%$ torsion loading at $60^{\circ} \mathrm{C}$ have a lifespan lower than 1 hour and those tested at ambient temperature have a lifespan greater than 19000 hours.

- These global trends are observable on both resin systems which seems to indicate that they are not specific to one given material composition.

Torsion specifically loads the matrix, these results therefore highlight matrix's role in the creep rupture of composite materials. When torsion is added to the bending test, it superimposes a shear stress to the material subjected to either traction or compression and accelerate the creep rupture process.

The model proposed in this paper will specifically interest on a combined traction torsion loading and the influence of torsion load level on the long term durability of composites with viscoelastic matrix.

\section{Creep and creep rupture of UD composites, literature overview}

Creep rupture was observed in various configurations from model composites to large-scale structures. Prestressed concrete tendons and rebars (Ceroni et al. [12]), pultruded frame (Bank and Mosallam [13]) as well as rod composite samples (Kim et al. [14]) and model laboratory samples with a few fibers (Zhou et al. [15]) failed at a stress level below their initial strength. Subject to constant load, the material undergoes a progressive damage: successive fiber breaks occur. Initially sparse, they tend to form a cluster just before composite's failure. This cluster formation was also experimentally observed in the elastic case through high resolution CT in (Scott et al. [16]). This phenomenon is due to the time-dependent behavior of composite's components, especially the matrix. Indeed, fibers' creep can generally be neglected in comparison with matrix's, even if their mechanical characteristics are still evolving and even if they are vulnerable to corrosion which causes the diminution of their strength and potentially rupture(Numerous defects are present in glass fibers [17] and several mechanisms creating these defects [18])

As mentioned before, the load bearing component in unidirectional fiber reinforced polymers (referred to as UD FRP in what follows) are fibers but according to matrix's behavior, long-term performances of the whole composite may differ tremendously. Indeed, matrix is responsible for load transfer between fibers. Due to its viscoelasticity, load transfer lengths are increasing in time thus provoking general creep of the composite, and deferred fiber ruptures that could eventually result in composite's failure. Matrix creep depends on stress level and can be modified by environmental conditions such as humidity or temperature. In some conditions matrix located between adjacent fibers can yield, creating inelastic zones where matrix's load transfer capacity is altered [19]. Matrix's yielding is related to its intrinsic properties (yield strength, 
stiffness...) but also to fiber spacing and globally applied load. The state of the fiber-matrix interface is determinant in the load transfer process. Interface treatment and fiber coating are therefore important factors in long-term durability of the composite and widely influence the debonding sensibility. To understand the debonding process, two aspects need to be treated: interface strength criterion that is appropriate to be applied and the residual stress state at the interface after debonding. Fibers mechanical (production and handling) and chemical treatment has a primary influence on the matrix-fibers adhesion. Among these treatments, the choice of sizing in a given fibers-matrix configuration is one of the most decisive. A study of the damage micromechanisms around a broken fiber with different sizings embedded in epoxy matrix [20] demonstrated that the optimal interface is not necessarily the strongest. As a matter of fact when the interface is too weak, the stress transfer is not performed properly: fiber ineffective lengths are too important which weakens the composite. On the other hand when the interface is too strong, stress from broken fibers is transferred to the intact neighbors in a very localized manner. As a result of this stress concentration, the strength of the material is diminished. An interface enabling a good stress transfer but able to absorb fiber break energy through debonding and cracking is a good compromise.

Debonding is not the only inelastic behavior that can be observed at the fiber-matrix interface. Plasticity, where matrix has yielded and irreversibly sheared, can also occur. Experimental observations of graphite fibers embedded in an epoxy matrix, presented in [21] and [19] as well as [22] suggest that the type of inelastic zone obtained depends on the fiber spacing, material system and fiber surface treatment. With low fiber volume fractions, slip occurs, whereas with elevated fiber volume fractions, matrix tends to yield. Under quasi-static conditions, inelastic zones initiate and grow when a critical shear stress is reached. The remaining yield shear stress or frictional shear stress is lower than this critical value.

The mechanism of creep rupture at the fibers' scale is generally admitted to be the following:

1. When load is applied to the material a few fibers may break instantaneously even if the applied load is lower than the global strength of the material. This is due to the fact that fiber's strength is not constant on its entire length: weakening defects are randomly located on the fiber.

2. Around fiber breaks matrix is locally loaded in shear. This shear stress enables the broken fiber to recover progressively its initial load far from the broken site. Close to the rupture site the load initially supported by the broken fiber is redistributed to its surrounding neighbors also via matrix's shear.

3. Even if a constant global load is applied to the material, several time-dependent phenomena take place. The first one is the time evolution of the fibers' strength. Preexisting defects in the fibers can be activated by the applied load (lower than fiber's strength). Stress corrosion cracking can then occur (in the case of ceramic fibers) leading to fiber's progressive rupture or at least to the reduction of fiber's strength.

4. The second time-dependent phenomenon is due to the viscoelasticity of the matrix. Around fiber 
breaks, matrix shear stress relaxes in time, increasing stress recover length and broadening overstress profiles on the neighboring fibers.

5. While broadening, overstress profiles due to several fiber ruptures on an intact fiber will overlap thus leading to locally increasing axial fiber stress which might result in a new break.

6. Inelastic zones around the fiber break tend to develop depending on fiber spacing and applied load intensity. Fiber-matrix debonding and/or matrix plasticity can occur modifying load transfer length.

These basic micromechanical mechanisms of creep rupture have been identified through various experimental studies and modelled using different approaches (Mishnaevsky and Brøndsted [23]). Shear-lag models are one of the most often used approaches as they capture the main features of stress redistribution in a composite with broken fibers and are easily implemented [15, 19, 21, 24-39]. These analytical models enable to understand the damage evolution process in a composite subject to a loading. In shear-lag models fibers are 1D tension-spring elements surrounded by matrix. Generally matrix's axial stiffness is neglected and the entire axial load is carried by the fibers whereas matrix supports exclusively shear stresses (a detailed discussion of classical shear-lag assumptions was made in 1997 by Nairn [30]). When a fiber breaks, its load is redistributed to its immediate neighbors via matrix's shear (Local Load Sharing models, LLS) or to all of the remaining fibers (bundle models or Global Load Sharing models, GLS).

Cox in 1952 was the first one to develop the load transfer model around a single short fiber imbedded in an elastic matrix (Cox [24]). Then Hedgepeth developed an elastic shear-lag model in its presently well-known form. In his technical note of 1961, he gave the static and dynamic solution to the problem of $n$ aligned fiber breaks in an elastic matrix (Hedgepeth [25]). Rosen in 1964 introduced stochastic distribution of fibers' strengths into the shear-lag model. His experimental observations also contributed to the understanding of FRP's strength and failure (Rosen [26]). In his model the ineffective fiber length after its failure is calculated using shear-lag analysis. Both the elastic and elastic-plastic behavior of the matrix are considered. Then a bundle model is combined with weakest-link statistics to estimate the rupture stress of the composite given its constituent's characteristics. In his model the effect of stress concentration due to fiber breaks was ignored (as GLS model was used) but in 1968 Zweben introduced this effect in the Rosen's model (Zweben [27]). Van Dyke and Hedgepeth [28] in 1969 investigated the influence of fiber-matrix debonding and matrix yielding on the stress concentration factor due to a single fiber break in a finite and an infinite lamina, both in $2 \mathrm{D}$ and $3 \mathrm{D}$ fiber arrangements.

Staggered fiber breaks bring new difficulty that was solved with the development of Break Influence Superposition (first introduced by Sastry and Phoenix [29] in 1993) and alternatively the use of Green's functions (Xia, Curtin, and Peters [40]).

Influence of fiber-matrix slipping, uneven fiber spacing and matrix axial stiffness were investigated in 1999 by Landis and McMeeking [33] in the elastic case. These models allowed the evaluation of composite's static 
strength. Other models aimed specifically at simulating the micromechanism of composite's degradation and rupture $[34,36]$. Alternatively to the shear-lag models, 3D FE models were also developed. The model developed in Blassiau et al. [41] takes into account matrix's axial stiffness and fibre-matrix debonding in the elastic case. Experimental and simulation results were compared in Scott et al. [42] for a composite with a fiber volume fraction of $60 \%$. Another more recent comparison between experiments and simulations using shear lag model and stochastic fibers strength were provided through the case of $2 \mathrm{D}$ bamboo fiber reinforced polymer matrix composites [43].

Recently concerns about long-term durability of composite materials subject to constant load have arisen. This involves taking into account matrix's viscosity. Several models consider a viscoelastic matrix. In [44] the evolution of overstress profiles with $n$ aligned fiber breaks in a viscoelastic matrix was investigated. The evolution of the stress state in a composite with a unique fiber break and a viscoelastic matrix was studied in [35] using two different models for matrix's behavior. In 1998 Beyerlein et al. [31] studied the time evolution of the stress field in a 2D unidirectional composite with several staggered fiber breaks. In Beyerlein et al. [19], Zhou et al. [21], Koyanagi et al. [38] inelastic zones of fiber-matrix debonding were also modelled. These models give a good approximation of composites with large fiber spacings. The finite element model presented in [41] is further developed to take into account matrix's viscoelastic and plastic behaviors in Blassiau et al. [45]. Finally, we can also report a recent model developed by Monfared and Mondali [46], in order to study the creep strain rate in short fiber unidirectional composite.

The main drawback of shear-lag models is their computation time for large-scale composites. When the strength of a large-scale sample is to be evaluated, multi-scale models can be employed such as the one proposed by Xia, Curtin, and Peters [40] or Guedes, Morais, Marques, and Cardon [47]. In Mahesh and Phoenix [48] a shear-lag model is used to predict long-term damage evolution of a small-scale composite, then weakest link scaling is used to determine the behavior at a larger scale. Finite Element models have also been successfully combined with the multi-scale models to model the rupture of macro-scale composite structures (Blassiau et al. [49]). Micro-scale models such as shear-lag models provide with damage evolution laws that can be integrated in continuous mechanics models such as the one proposed by Nedjar [50], or more recently [51], for the evaluation of long-term behavior of composite structures. Macro-scale models that do not account for phenomena on the fibers' scale need the results from such micro-scale models to identify long-term behavior of the material resulting from matrix relaxation and fiber breaks. Another way to make a link between micro and macroscale is proposed by Na et al. [52]. Using statistical approach and the stress concentration factor relative to the interface shear strength and the geometry of surroundings obtained by 3D FEM analysis, they are able to predict the strength of a given composite.

Under steady-state creep conditions, where interfacial shear stress relaxes in time, inelastic zones still initiate and grow which means that a constant stress criterion is no longer appropriate and a strain-based 
criterion is therefore more realistic. It was shown in [21] that the interfacial shear strength decreases inversely with the square root of matrix's compliance.

In this same article a single fiber shear-lag model is developed. Its results are compared to experimental observations obtained using micro-Raman spectroscopy under steady-state creep conditions. In the experiments, several fiber volume fractions and strain levels are tested. The developed single fiber shear-lag model assumed a shear strain-based criterion for debonding propagation. Comparison with experimental data confirmed the applicability of this criterion.

In another experimental study by Koyanagi et al. [38], a single-fiber composite is tested under constant strain duration. In order to do so, a composite, consisting of carbon fibers embedded in a vinylester resin, with a very low fiber volume fraction is used. Then micro-Raman spectroscopy is applied to assess fiber axial strain. Shear-lag approximations, based on the model developed by Beyerlein et al. [19], then enable to derive matrix shear stresses and interpret the experimental results. But, Instead of assuming a constant frictional/yielding shear stress in the fiber-matrix debonded region as it was done in [19], this condition is modified in order to take into account Coulomb's friction influenced by the time-dependent relaxation of the matrix?s radial stress. Moreover, instead of using a strain-based criterion, a stress-based debonding criterion, also assumed to be enhanced by Poisson's contraction stress around a fiber break, was implemented in the analysis. Therefore this critical stress decreased in time as a result of matrix's relaxation. An empirical expression of the debonding shear stress is derived. Finally, using this model and the described modifications, an expression of the ineffective fiber length is derived. Then a GLS (global load sharing) model is used to derive the time-dependent degradation of the tensile strength of the whole material. Experimental and analytical results being in close agreement, authors conclude that the degradation of tensile strength under constant strain is caused by the degradation of the interfacial stress-transfer capacity.

In a subsequent work this GLS model is further developed [53]. In the GLSV (Global Load Sharing Viscoelastic model), a time-dependent frictional stress is derived. It is related through Coulomb's law to the radial stress in the matrix, which in turn is related to the axial stress in the matrix through Poisson's effect. Indeed, compression occurs at the fiber-matrix interface around a broken fiber: when elongation is applied to the material, the material shrinks in the transverse direction. Around the fiber break, the fiber is unloaded, its longitudinal strain is therefore low compared to that of the matrix. Since the unloaded fiber does not shrink in the radial direction, it applies a compressive load on the surrounding matrix.

Following experimental investigations $[54,55]$ showed that a distinction has to be made between the "pure" interfacial strength, independent of time and temperature and the apparent interfacial strength enhanced by the previously described Poisson's ratio effects. These effects relax in time which leads to an apparently diminishing interfacial strength. However matrix's strength is time-dependent which could cause premature loss of fiber-matrix cohesion. FEM simulations [56] including this representation of the fiber-matrix interface were implemented and compared to micro-Raman spectroscopy results on single-fiber 
composites. A good agreement was found between the model and experimental observations confirming model's validity.

In previously described models, time-dependent debonding was simulated in low fibers volume fraction composites where interactions between fiber breaks are neglected. In the elastic case, a model presented in [57] (a development of 1995's model introduced in [58]) combines the effects of debonding and stress concentrations due to the local load sharing between the fibers. Time-dependent effects were not taken into account in this model.

It is the objective of the present paper to combine developments of this literature and propose a model that would include time-dependent effects of local load sharing as well as time-dependent debonding and friction thus extending the application of viscoelastic shear-lag models to the case of unidirectional composites with elevated fiber volume fractions. In order to do so, the model introduced in Beyerlein et al. [58], which allows to treat debonding in the elastic case is developed in the viscoelastic case and is combined with the model described in Beyerlein et al. [31] devoted to the time evolution of stress redistribution in a composite with several cracks and without debonding. A shear strain-based debonding criterion and a time-dependent friction are introduced in a similar way to that presented in Zhou et al. [21] and Koyanagi [53], linking it through Coulomb's law and the Poisson's effect to the axial stress in the matrix. Finally, these numerical developments proposed in this paper predict an increase of the lifespan of the material subjected to combined traction-torsion loading. A future model should interests in combined compression-torsion loading to explain the decrease of lifespan observed in combined bending-torsion loading.

\section{Theoretical basis of the model}

In order to model fibers' breaks and debondings evolution in time in a unidirectional composite material subjected to a constant traction load with, linear elastic brittle fibers, frictional interfaces and a linear viscoelastic matrix , a shear-lag type approach highly based on the models of Beyerlein et al. which are thoroughly presented in Beyerlein et al. [31] and Beyerlein et al. [58] is used. The first step in solving this problem is to model the evolution of stress distribution for a given rupture pattern.

Considering an infinite unidirectional composite material with elastic fibers imbedded in a viscoelastic matrix subject to constant tension load applied in the longitudinal direction, they proposed a mechanical solution involving multiple fiber breaks and delaminations. This defines the general problem P1, figure 3. For that, they used a superposition technique described in Beyerlein et al. [31] for the study of fiber breaks accumulation and in Beyerlein et al. [58] for the description of the debonding. The general problem, here P1, can be seen as a combination of two subproblems SP1 and SP2. The first one corresponds to a multi-damaged material where a unitary compressive load is applied at the tips of every broken fiber (sites marked with a -1 in the figure 3 , SP1), a given frictional stress ( $\tau$, figure 3 , SP1) is imposed in the debonded regions 
and zero load is applied at the far field. The second subproblem SP2 consists of an undamaged material subjected to a uniform stress state. The solution to the subproblem SP2 is trivial and one needs to find the solution to the subproblem SP1.

To obtain the solution to the general problem P1, these two solutions (SP1 and SP2) are superimposed. In the subproblem of the damaged material (SP1), the stress state in the material results from a weighted superposition of the individual influences of each fiber break site and matrix debonded region in the material. If fiber breaks are solely considered the stress state results from a weighted superposition of the influences of fiber breaks only. Each of which is a solution to the auxiliary problem A1, see figure 3, treated in Beyerlein et al. [31]. $K_{i}$ represents the influence of each fiber break on the whole composite. Among other results of this analysis, shear stress in the matrix is obtained. In order to take debonding into account, the idea, introduced in [58], is to modify this shear stress profile in order to locally obtain a uniform frictional stress in the debonded region. This shear stress is then used to rectify the shear stress profile in the debonded region obtained around a broken fiber. This procedure is represented in the figure 4: a fiber break creates a shear stress concentration in the neighboring matrix (red $\tau$ curve). If the associated shear strain is greater than the critical debonding shear strain, debonding occurs. In the debonded region a uniform frictional stress is established (blue dotted curve). In order to impose this shear stress, the shear stress profile due to the fiber break is modified by superimposing to it the solution to the auxiliary problem A2.

Firstly, to solve A1 and determine the stress distribution due to an isolated fiber break Beyerlein et al. [31] the local equilibrium equation of a fiber's element $n$ is consider. It can be written as given in the equation (1). $\sigma_{n}(x, T)$ is the axial stress in the fiber $n$ at the longitudinal coordinate $x$ and at time $T$. $\tau_{n}(x, T)$ is the shear stress in the matrix band $n$ and $h$ is the fiber's diameter.

$$
\frac{\partial \sigma_{n}(x, T)}{\partial x}+\frac{\tau_{n}(x, T)-\tau_{n-1}(x, T)}{h}=0
$$

A perfect bonding between the matrix and fibers is here assumed, fibers are considered to be linear elastic, and matrix is assumed to be linear viscoelastic and its shear stress depends on the history of the shear strain, via a the matrix's relaxation function $G(T)$ and the charcateristic time $T_{c}$. A power law expression of the following matrix's creep function $J(T)=\frac{1}{G(t)}$ is used.

$$
J(T)=J_{e}\left(T / T_{c}\right)^{\alpha}
$$

To make variables and equations dimensionless, some normalization constants are introduced as $\xi$ the normalized longitudinal coordinate $\xi=\frac{x}{\sqrt{\frac{w E_{f} A J_{e}}{h}}}$ and $t$, the normalized time $t=\frac{T}{T_{c}}$. It leads to the following differential equation (3) (Beyerlein et al. [31]).

$$
\frac{\partial^{2} U_{n}}{\partial \xi^{2}}+\int_{-\infty}^{t} G\left(t-t^{\prime}\right) \frac{\partial}{\partial t^{\prime}}\left(U_{n+1}-2 U_{n}+U_{n-1}\right) d t^{\prime}=0
$$




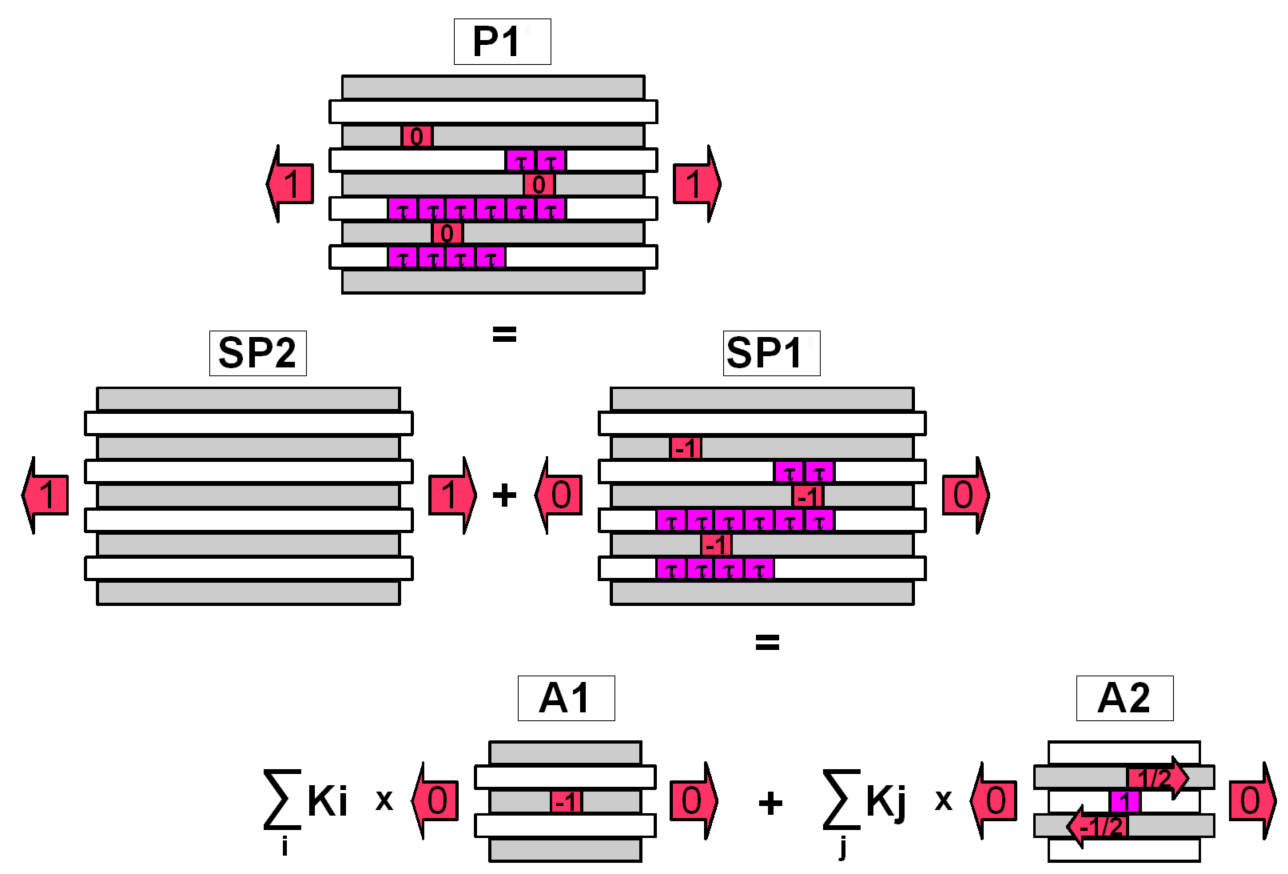

Figure 3: General problem P1 as a superposition of the subproblems SP1 and SP2. The subproblem SP1 is obtained through a weighted superposition of the auxiliary problem A1 and A2

which gives a relation between normalized axial displacements of the $n, n+1$ and $n-1$ fibers.

When multiple fiber breaks are considered, each fiber break taken individually will cause over-load on surrounding fibers including other fiber break sites. And yet, at all time step, zero stress condition on the fiber tips must be satisfied in the general problem P1. Which is equivalent to have unit compressive load at each fiber break site in the subproblem SP1. Therefore the influence stress fields due to individual fiber breaks (resulting from A1) must be multiplied by a time-dependent coefficient (called weight function $K_{i}(t)$ ) and the solution of the general problem P1 of an infinite lamina subjected to unit traction at the far field and zero stress at the broken sites may be given.

To take debonding into account, the subproblem A2 has to be solve. It was proposed in Beyerlein et al. [58] for the elastic case. An improvement is developed here, since a matrix's viscoelastic behavior is taken into account for our simulations.

This auxiliary problem A2, consists of an infinite undamaged lamina where two fiber elements surrounding a central matrix element are subjected to equal and opposite traction loads (marked with $\pm 1 / 2$ in the figure 3 , A2). This load state results in a locally applied unitary shear stress on the central matrix element (1 on the figure $3, \mathrm{~A} 2$ ). The auxiliary problem can be summarized by the diagram presented in the figure 5 . In 


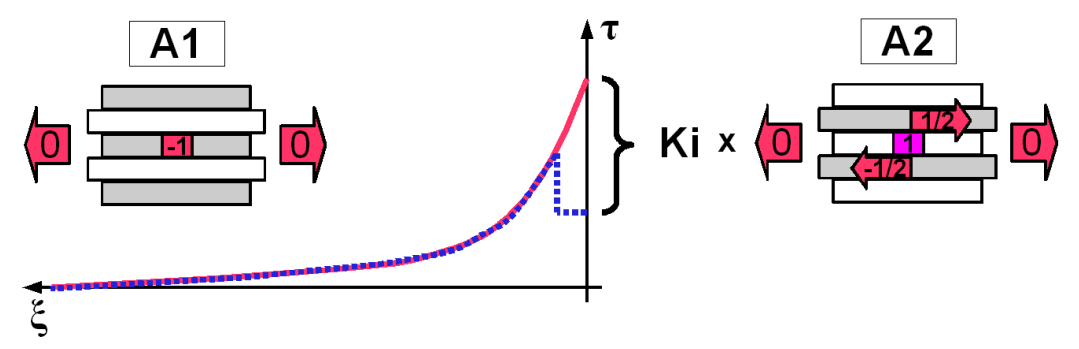

Figure 4: Modification of the matrix shear stress profile to take debonding into account through the use of the superposition technique.

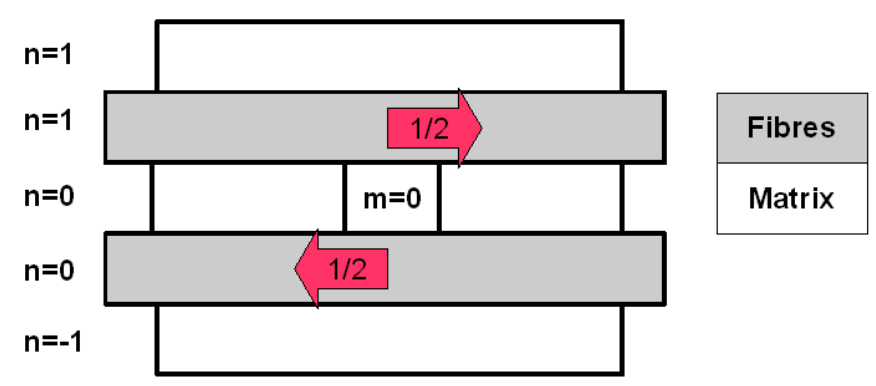

Figure 5: Auxiliary problem A2: infinite undamaged lamina where two fiber elements surrounding a central matrix element are loaded with equal and opposite loads.

this figure $n$ gives the fiber or matrix band number and $m$ gives the fiber/matrix element number in the longitudinal direction. In this problem a central matrix element with $m=0$, located in the matrix band number 0 is subjected to a shear stress resulting from axial forces applied to the fiber elements surrounding the considered central matrix element (on the figure 5, 1/2 and $-1 / 2$ applied to fibers number 1 and 0 respectively).

Remains of fibers are elastic and brittle, and the solution to the A2 problem in the elastic case can be found in Beyerlein and Phoenix [57] and Beyerlein et al. [58]. For the purpose of our work this approach was developed to the viscoelastic case in a very similar way that for the isolated fiber break in [31]. It leads to the same differential equation (3) on normalized axial displacements of the $n, n+1$ and $n-1$ fibers but with a different set on boundary conditions.

Finally, when multiple debonded matrix element are considered, each of it will affect on the surrounding fiber and matrix elements including broken and debonded one. Then, in the same way as the subproblem SP1, the influence stress due to the debonding matrix element must be multiplied by a time-dependent coefficient (called weight function $K_{j}$ ). We detailed nether the calculations of solutions of A1 and A2 problem nor ones of weigth functions $\left(K_{i}\right.$ and $\left.K_{j}\right)$ in this paper since they are quite complex and may be find combining the methodology described in Beyerlein and Phoenix [57] and Beyerlein et al. [58] with 
the viscoelastic development used in [31] for A1 problem (isolated fiber break). The next part details the strategies to simulate the progressive damage of a composite with fiber breaks and delaminations. In particular, we will describe in 5.2 the way to take into account viscoelastic effect on debonded part, in a similar way than Koyanagi [53].

\section{$5 \quad$ Progressive time-dependent damage}

In order to compute the time-dependent damage of the material including fiber breaks and debonding, following steps are required:

1. A sample is generated, that is random values of fibers strengths are allocated to every fiber element.

2. At the first time step, the load is uniform as no damage sites are present. The axial stress in the fibers and shear strain in the matrix are computed.

3. The most overloaded element is determined. It could either be a fiber element for which the applied axial stress is compared to its attributed strength or it could be a matrix element whose applied shear strain is compared to the constant shear strain debonding criterion (see following section 5.1).

4. When an element breaks the stress and strain state of the material is recalculated including this break,

5. Using this new stress and strain state, the most overloaded element is determined again.

6. The process is repeated until no additional broken element needs to be generated for the stress/strain state obtained for this given time step, then time increases by one step.

To increase the computational speed in the simulations presented in this paper, several most overloaded matrix elements debonded at each iteration instead of one at a time (fiber breaks were still treated individually).

\subsection{A shear-strain debonding criterion}

When initially undamaged material is subjected to constant strain (when a constant axial stress is applied to the elastic fibers at the far field), fiber breaks occur. Around these fiber breaks matrix is subjected to shear. As matrix's shear stress relaxes in time, corresponding shear strain increases. In the present model, when this shear strain reaches a limit value $\gamma_{l i m}$, constant in time, debonding occurs. In the debonded region, a frictional shear stress is imposed in the fiber-matrix interface (represented by the matrix elements). It is done through the superposition of the time-dependent solution found in the auxiliary problem A2, described previously, to the solution of a material where multiple fiber breaks exist. This solution is also timedependent and matrix's strain keeps increasing in time. When new matrix elements reach the constant shear-strain criterion, debonding progresses. 


\subsection{Time-dependent frictional shear stress}

When debonding occurs in a matrix element, a given frictional stress is imposed on it. This frictional stress is taken time-dependent according to the literature. The article Koyanagi [53] gives a methodology to take into account the influence of the matrix's relaxation on the value of the frictional shear stress. This methodology will be adapted to the presently developed model. Originally this methodology was applied in a Global Load Sharing context which implies certain hypothesis and results in approximations in the present model.

- Frictional stress is considered to depend entirely on the radial compressive stress in the matrix (due to Poisson's effect) and the frictional coefficient of the fiber-matrix broken interface.

- The value of this compressive stress is dependent on the relative shrinkage of the matrix and the fibers as well as fibers arrangement and volume fraction.

- The GLS model ignores the localization of the stress redistributions due to fiber breakage. Indeed in the vicinity of a broken fiber, the following mechanism is assumed: the composite material surrounding the broken fiber is subjected to traction and as a consequence it shrinks in the radial direction; the broken fiber itself is unloaded, therefore it does not shrink in the radial direction imposing on the surrounding matrix a compressive stress. If the Local Load Sharing scheme is assumed, the localization of the overload on the neighboring fibers should, in theory, lead to their greater radial deformation. For simplicity reasons, in the present model, the effects of this localization will also be ignored in the calculation of the value of the resulting compressive radial stress. The present model could be further developed by including this feature.

A frictional coefficient links the radial compressive stress and the frictional stress in the debonded region as shown in the equation (4):

$$
\tau_{f r}(t)=\mu \sigma_{r}(t)
$$

Where $\tau_{f r}$ is the frictional shear stress in the matrix's debonded element, $\mu$ is the frictional coefficient and $\sigma_{r}$ is the radial compressive stress at the matrix-fiber interface.

It will be assumed that the radial compressive stress is related to the matrix's far field longitudinal stress as expressed in the equation (5):

$$
\sigma_{r}(t)=\nu \sigma_{m}(t)
$$

Where $\nu$ is a specific Poisson's ratio. In the present study, it will be equal to that of the matrix. The matrix is viscoelastic, the relationship between its axial stress and strain can be written as:

$$
\sigma_{m}(t)=\int_{-\infty}^{t} E_{m}\left(t-t^{\prime}\right) \frac{d \varepsilon_{m}\left(t^{\prime}\right)}{d t^{\prime}} d t^{\prime}
$$


In the present model, a constant in time axial fiber stress is applied. Because fibers are elastic, this implies a constant strain in the fibers at the far field. At the far field fibers and matrix are undamaged, their axial strains are therefore equal. This approximation simplifies the previous equation in the following manner:

$$
\begin{aligned}
\sigma_{m}(t) & =E_{m}(t) \varepsilon \\
\varepsilon & =\frac{\sigma_{f}}{E_{f}}
\end{aligned}
$$

Where $\varepsilon$ is the imposed composite strain. As for the shear behavior, an incomplete power law will be used for matrix's axial viscoelastic behavior, equation (8):

$$
E_{m}(t)=\frac{E_{0 m}}{\left(\frac{t}{T_{c}}\right)^{\alpha}}
$$

Combining equations (4) to (8), the time-dependent expression of the frictional stress is obtained in the equation (9):

$$
\tau_{f r}(t)=\mu \nu \frac{\sigma_{f}}{E_{f}} \frac{E_{0 m}}{\left(\frac{t}{T_{c}}\right)^{\alpha}}
$$

In these simulations, at each time step, the frictional shear stress imposed in the debonded part $\tau_{f r}(t)$ is then recalculated.

\section{Basic stress transfer phenomena}

In this first analysis, a simple case of an infinite lamina with one central fiber break is presented. A traction load is applied to the material. A shear deformation peak results from this load in the very vicinity of the fiber break. The excessive shear strain causes debonding. In time, as matrix relaxes, the debonded region progresses. In the debonded region, an uniform frictional shear stress is imposed. According to the previous sections, this frictional stress diminishes in time. In 6 and 7 , material data used in the simulation is given in 3 in part 7 fixing Ge to 4.5GPa. Moreover, stress value are normalised to make them dimensionless as proposed in [31], i.e, the fiber axial stress is normalized by its value without fiber break $p^{*}$ and the matrix shear stress is normalized by $\frac{p^{*}}{E_{f} * A * h * w * J_{e}}$. On the figure 6(a), the time evolution of the shear stress in the matrix surrounding the broken fiber is represented. It can be seen that the debonded region extends in time (shear stress peaks move apart) while the frictional stress slightly diminishes (on the figure 6 (a) the constant shear stress in the middle debonded region is decreasing in time). On the figure 6 (b) the axial stress in the broken fiber and its first intact neighbor as well as their evolution in time are shown. The overstress profile evolves in time progressively presenting several maxima instead of one, as it would be the case without debonding. Another difference with the case without debonding is the fact that in time the overstress of the first intact fiber in the very vicinity of the fiber rupture is decreasing instead of remaining constant. This is due to the relaxation of the frictional stress and the progressive global load sharing in the 


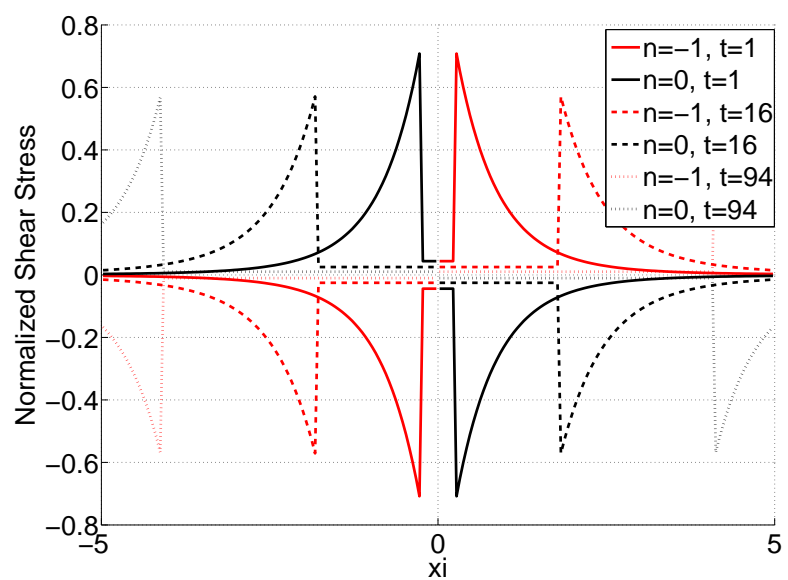

(a) Matrix shear stress around the broken fiber

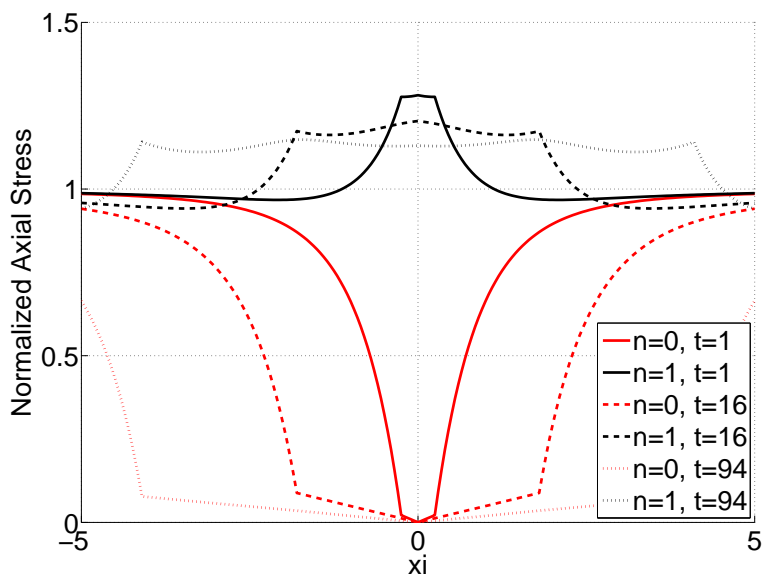

(b) Axial stress in the broken fiber and its first intact neighbor

Figure 6: Influence of a broken fiber on its immediate surroundings including debonding

material. The load is progressively more equally distributed among the remaining intact fibers instead of being mainly supported by the first intact fibers.

The stress redistribution scheme is also modified on the following neighboring fibers. Figures 7 (a) and 7 (b) show the overstress profiles on the 1st to 4th intact fibers next to the broken one, respectively at the first and third time step. When time progresses, instead of presenting only one localized stress peak, the overstress profiles tend to a more uniform distribution on the whole overloaded region of the intact fiber. This is in significant contrast with what was observed in simulations ignoring debonding (figures 7 (c) and 7 (d) present these results) where stress redistribution was highly localized around the fiber break and dominantly concentrated on the first intact neighbor, a situation persistent in time. 

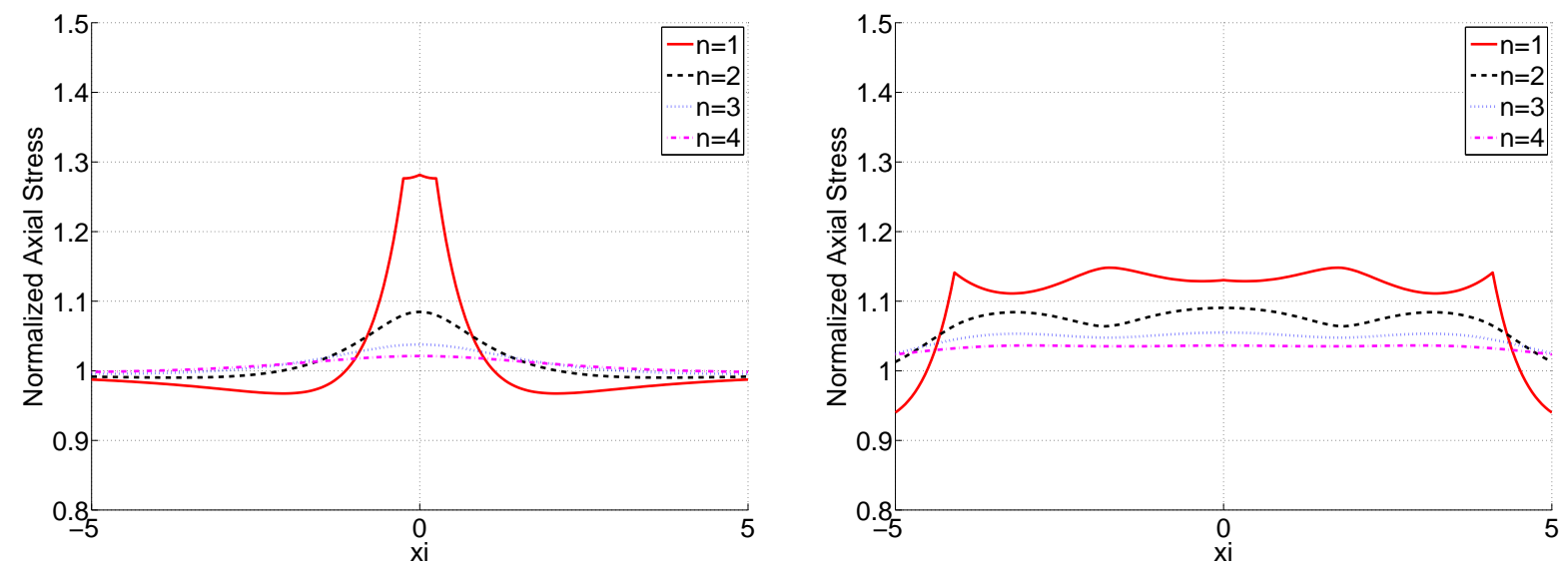

(a) Axial stress in the neighboring intact fibers at the first (b) Axial stress in the neighboring intact fibers at the 3rd time step (elastic response) when debonding occurs time step when debonding occurs

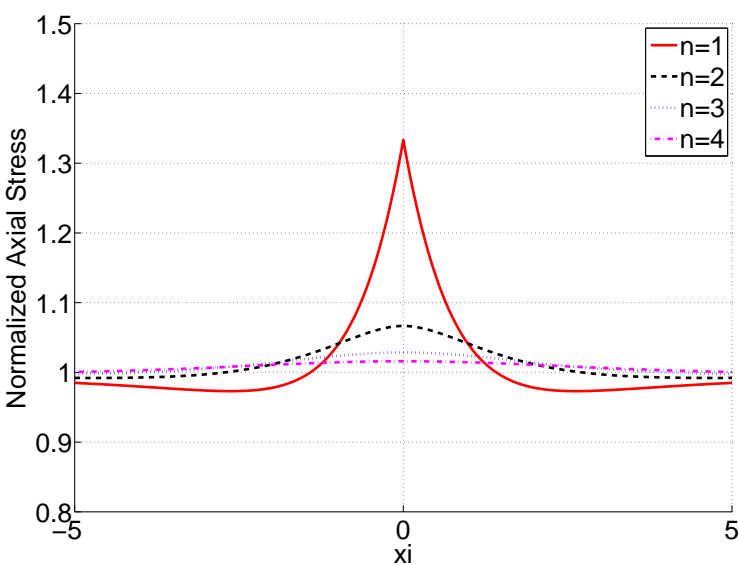

(c) Axial stress in the neighboring intact fibers at the first time step (elastic response) without debonding

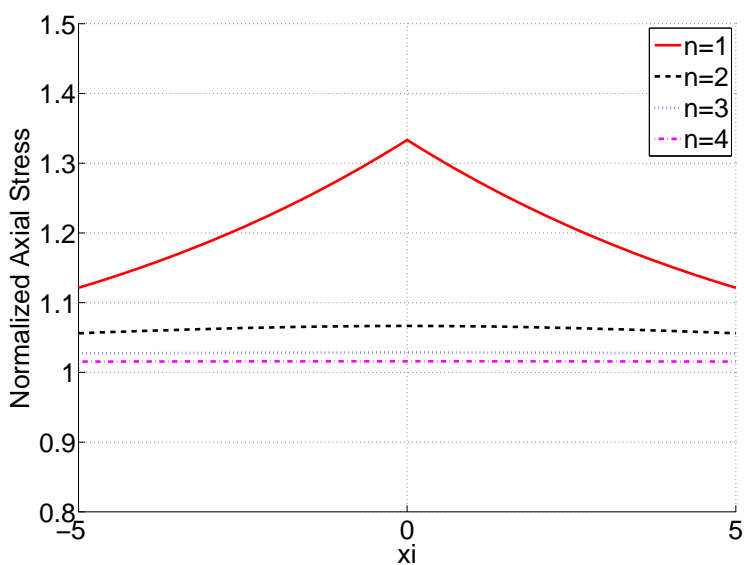

(d) Axial stress in the neighboring intact fibers at the 3rd time step without debonding

Figure 7: Influence of a broken fiber on its 1st to 4th intact neighbors. Comparison between the situations with and without debonding

Maximum overstress factors are summarized in the table 2. The overstress factor (OSF) equals the stress in the fiber element divided by the axial far field stress applied to the material $\left(O S F=\sigma_{f} / \sigma_{0}\right)$. When $O S F>1$, the fiber supports a stress greater than in the unscathed composite. On the other hand when $O S F<1$, the fiber is partially or completely unloaded. The Maximum OSFs are compared to those obtained when debonding is not accounted for and with results taken from a $3 \mathrm{D}$ FE model presented in Blassiau et al. [59].

When debonding is not modelled, the OSF is respectively higher for the first intact neighbor and lower 


\begin{tabular}{lcccc}
\hline Fiber's number & $\mathbf{1}$ & $\mathbf{2}$ & $\mathbf{3}$ & $\mathbf{4}$ \\
\hline OSF without debonding (constant in time) & 1.333 & 1.067 & 1.029 & 1.016 \\
\hline OSF with debonding at the first time step & 1.282 & 1.085 & 1.038 & 1.021 \\
\hline OSF with debonding at the third time step & 1.148 & 1.09 & 1.055 & 1.036 \\
\hline OSF without debonding at the first time step, taken from [59] & 1.084 & & & \\
\hline OSF with debonding at the first time step, taken from [59] & $1.13-1.16$ & &
\end{tabular}

Table 2: Maximal OSF of successive broken fiber's intact neighbors

for the following neighbors 2,3 and 4 when compared to the OSF obtained with debonding. This shows that with debonding, load sharing becomes more equal among neighbors approaching a global load sharing scheme. Comparing OSFs at the first time step and at the third time step shows that uniformization of stress over neighbors is further increased when debonding progresses. When comparing the present $2 \mathrm{D}$ shear-lag model's results with the results from a 3D FE model, it can be seen that in the elastic case without debonding, the OSF of the $3 \mathrm{D} \mathrm{FE}$ model is lower on the first intact neighbor. This can be partly explained by the differences between the $3 \mathrm{D}$ and $2 \mathrm{D}$ fiber arrangements. In the 3D case, there is more intact neighboring fibers supporting the overstress, therefore the amount of stress transferred to each of them is lower. Another observation should be made about the modification of the OSF when debonding occurs. The present model shows a reduction of the OSF whereas the 3D FE model shows an increase in the OSF. This might be partly explained by the fact that the shear-lag model does not take into account matrix's axial stiffness. In the FE model, stress supported by the matrix near a broken fiber would be further transferred to the intact fibers when debonding occurs as matrix becomes "inactive", this could result in the increase of the OSF. More importantly, in the present work, fiber-matrix debonding length is controlled by a shear strain-based criterion whereas in the FE model various debonding lengths are imposed. As FE simulations and the present work show, the longer the debonded region, the lower the overstress factor on the intact neighbor.

Results shown in the table 2 are specific to the interface strength used in this simulation. Lower interface strength would increase initial debonding span modifying the OSFs. Nonetheless this comparison gives the general trends of the influence of debonding on the stress transfer mechanism. These results confirm that a damageable interface may be more profitable to the material because it avoids stress localizations in the material (as shown experimentally in [20]). 


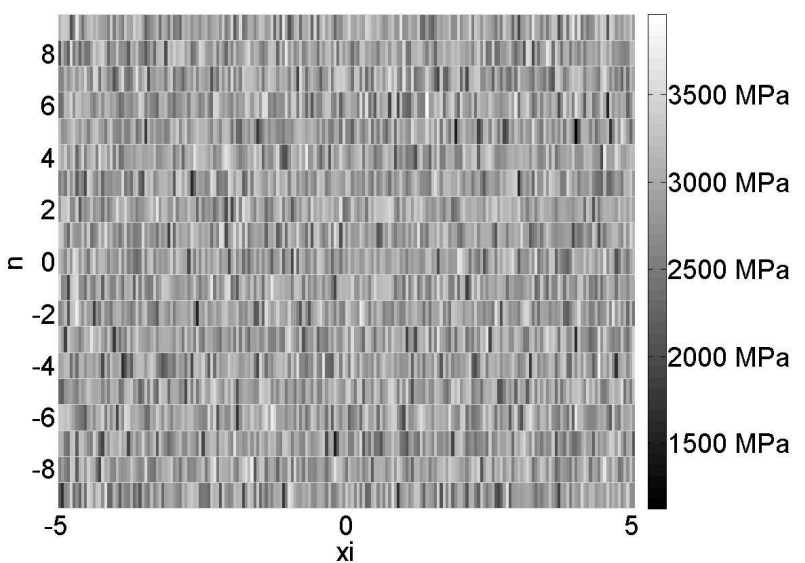

Figure 8: Strengths distribution in the fibers

\section{Progressive damage combining fiber breaks and debonding}

In this section a more complex case will be studied. A sample is first generated: random fibers strengths are allocated to element of discretized fibers. Further details on this procedure are given in chapter 2 of [11]. Then this sample is studied in the elastic case: its static strength is determined with and without taking into account the debonding. Next this same sample undergoes a creep test and the time-dependent damage (both fiber breaks and matrix debonding) is observed. The same creep conditions are then applied to this sample with a modification of the matrix's shear stiffness in order to assess its role in the time-dependent creep and damage. In a final subsection the influence of an additional shear stress (that could represent a torsion loading superimposed to a traction loading applied to the material) will be studied.

The sample consists of an infinite lamina where 19 fibers are allowed to break. Each fiber is divided into 201 elements. A randomly generated strength following the previously presented bi-modal Weibull probability law is assigned to every element [60]. The fiber volume fraction is taken equal to $54.2 \%$. The figure 8 represents the fibers strengths distribution of the specimen studied. Each horizontal band represents a fiber. Random distribution of flaws in the fibers can be noticed. Various parameter values used for this simulation are summarized in the table 3 .

The resin has mechanical properties of an epoxy resin (presented in [15]). In particular, the present value of poisson's coefficient has been deduced from young's modulus and shear modulus values given in [15]. Moreover, Fibers are glass fibers characterized in [60]. The parameter $\alpha$ characterizes matrix's creep behavior. The higher the value of $\alpha$, the more pronounced the matrix's creep is. Previously studied value of 0.5 for this parameter is rather high but certain polyester resins have this characteristic. Furthermore, 


\begin{tabular}{ll}
\hline Model characteristics & \\
\hline $2 \mathrm{~N}+1$ & 19 \\
$2 \mathrm{M}+1$ & 201 \\
normalized $d x$ & 0.05 \\
$V_{f}$ & $54.2 \%$ \\
loading & variable: 0 - 2800MPa (elastic case), 1910MPa (creep test) \\
shear loading $\gamma_{s h}$ & variable: 0 and $70 \% \gamma_{l i m}$ \\
\hline Fiber characteristics & \\
\hline$\sigma_{01}$ & $3200 \mathrm{MPa}$ \\
$m_{01}$ & 5.79 \\
$\sigma_{02}$ & $5110 \mathrm{MPa}$ \\
$m_{02}$ & 7.65 \\
$l_{0}$ & $10 \mathrm{~mm}$ \\
$q_{0}$ & 0.45 \\
$E_{f}$ & $74 \mathrm{GPa}$ \\
$r$ & $13.5 \mu \mathrm{m}$ \\
\hline Matrix characteristics & \\
\hline$G_{e}$ & $1.29 \mathrm{GPa}, 4.5 \mathrm{GPa}$ and $10 \mathrm{GPa}$ \\
$\nu$ & 0.3 \\
$T_{c}$ & $1600 \mathrm{~s}$ \\
$\alpha$ & 0.2 \\
$\gamma_{l i m}$ & $14.8 \%$ \\
$\mu$ & 0.5 \\
\hline
\end{tabular}

Table 3: Simulation parameters

elevated temperatures can intensify matrix's creep, increasing $\alpha$ 's value. The corresponding creep behavior of the composite material as a whole will be also more pronounced. Two additional values of $\alpha$ were used in our simulations, 0.2 and 0.048. 0.2 is a middle-range value for usual polymer matrixes and 0.048 characteristic of an epoxy resin (as in [15]. Some preliminary simulations were made without debonding: at 1500MPa with an $\alpha=0.5$ both stages of secondary and tertiary creep were observed. At the same level of applied stress with an $\alpha=0.2$ only secondary creep is observed and the final relative strain ratio equals $2 \%$. When $\alpha=0.048$ creep is almost imperceptible with final relative strain ratio of $0.2 \%$. Furthermore, we did not study the effect of the frictional coefficient $\mu$ 's value in this study. As ones can expect this parameter will 


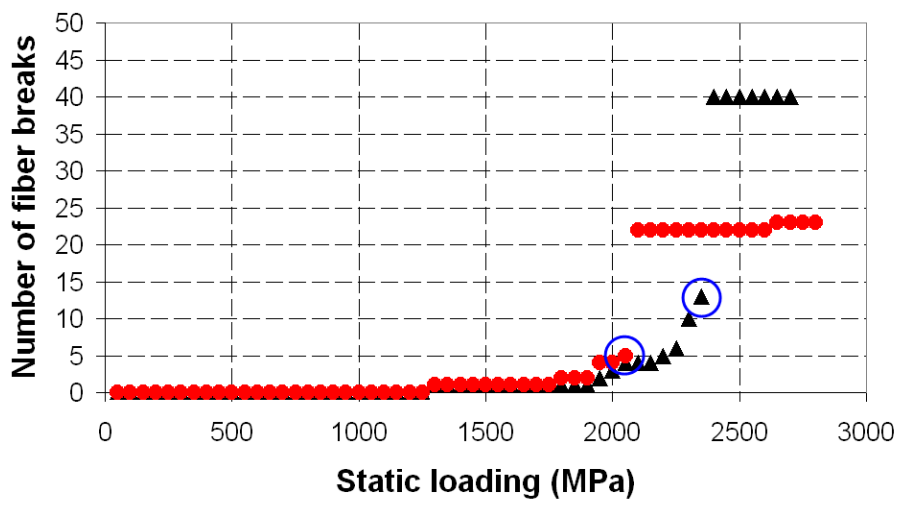

$\Delta$ without debonding $\bullet$ with debonding

(a) Number of fiber breaks versus applied load

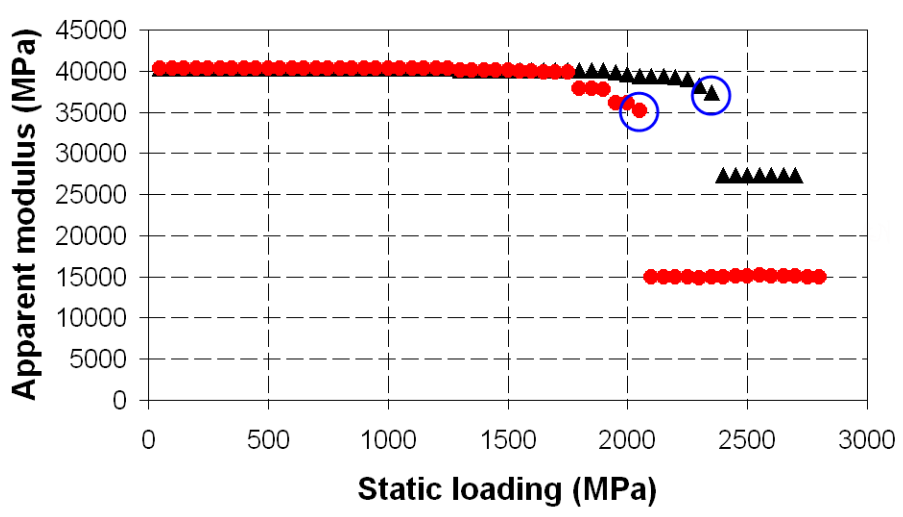

$\Delta$ without debonding $\bullet$ with debonding

(b) Apparent modulus versus applied load

Figure 9: Macroscopic damage versus applied load

be of interest, since the frictional stress on the debonded area varies in a linear fashion with it (equation 9). Nevertheless, most of the stress transfer is done by the pic of shear stress in the undebonded part. Then, a small modification of its value shouldn't severely change the global creep behaviour of the composite. In the following simulations paper $\alpha$ will be taken equal to 0.2 - as a middle-range value for usual polymer matrixes and $\mu$ will be set to 0.5 as given in Koyanagi [53].

\subsection{Static strength with and without debonding}

First, the sample is tested in static conditions. Increasing loading levels are applied in the elastic case. Corresponding damage is assessed. In particular the number of broken fibers is analyzed (fig. 9 (a)).

The point when its increase rate versus applied load increases abruptly is taken as the rupture (circled in blue on the figure 9 (a)). Corresponding applied stress is considered as the static strength of the material. 


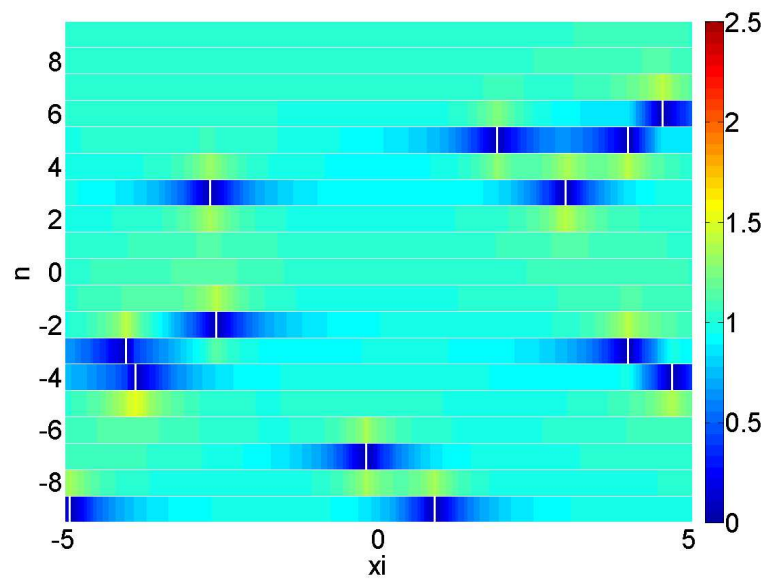

(a) $2350 \mathrm{MPa}-13$ fiber breaks

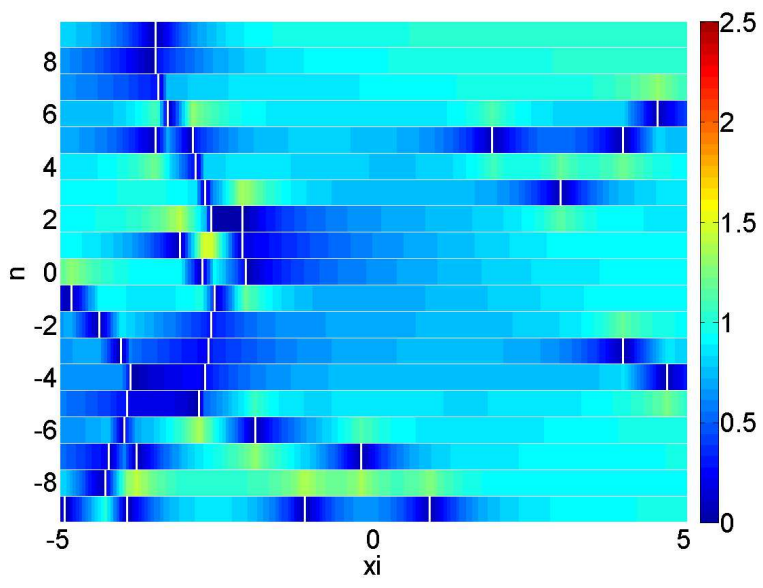

(b) $2400 \mathrm{MPa}-40$ fiber breaks

Figure 10: Damage patterns and overstress factors in the material without debonding

The sample is both studied in cases where debonding is taken into account and ignored. Thus the influence of debonding on the static strength of the material can be explored. In the figure 9 (a) the number of fiber breaks corresponding to each load level is shown. The influence of debonding is also assessed through calculation at each load level of the apparent modulus of the sample. These results are presented in the figure 9 (b). It is clearly visible that when debonding is taken into account the value of the static strength diminishes considerably. When debonding is ignored, the sample breaks when the axial far field stress exceeds $2350 \mathrm{MPa}$. When debonding is considered, the limit is $2050 \mathrm{MPa}$. In the same manner, the rupture moment of the material corresponds to a drop in the apparent modulus value (also circled in blue), as can be observed in the figure $9(\mathrm{~b})$.

In the following figures 10 and 11, damage patterns are presented for cases without and with debonding for loading levels preceding and slightly exceeding the rupture limit. In the figures 10 (a), 10 (b), 11 (a) and 11 (b) colors represent the level of the overstress factor for the fibers axial stress. In the figures 11 (c) and 11 (d) corresponding fiber breaks and debonded areas are represented.

It can be seen on these results that rupture occurring with debonding initiates with fewer fiber breaks (5 vs 13, see figures 11 (a) with debonding and 10 (a) without debonding) than when debonding is not considered. It can also be seen that when a fiber is broken, it is instantaneously unloaded almost on the entire length of the material through debonding (see figures 11 (a) and 11 (c)). The rupture pattern observed when debonding is modelled (figure $11(\mathrm{~b})$ ) is different from the one observed without debonding (figure $10(\mathrm{~b}))$. In the case with debonding fiber breaks are more scattered whereas without debonding they are mostly aligned. This is due to the fact that scattered fiber breaks linked by debonded areas (figure 11 (d)) 
form a unique break cluster. On the other hand when debonding does not occur, in order to be connected, fiber breaks need to be in the very vicinity of each other.

These static simulations show that debonding decreases the static strength of the material. In the elastic case matrix relaxation has not yet taken place. This means that load sharing between intact neighbors of a given broken fiber is still uneven: the first intact neighbor has a greater overstress factor. Due to the debonded region, this first intact neighbor is almost evenly overloaded on the whole length of the debonded region. Flaws on this intact neighbor can therefore be easily reached by the overstress leading to a new fiber break locate away from the initial fiber break causing the overstress. Debonded regions therefore connect fewer existing fiber breaks thus forming a macro-cluster. When debonding does not occur, overstress profiles are more localized, creating a peak of overstress in the very vicinity of the fiber break. A new fiber break occurs only if a flaw in the first intact neighbor is located immediately in this small overloaded region. Therefore higher loads are necessary to trigger global rupture of the material. 


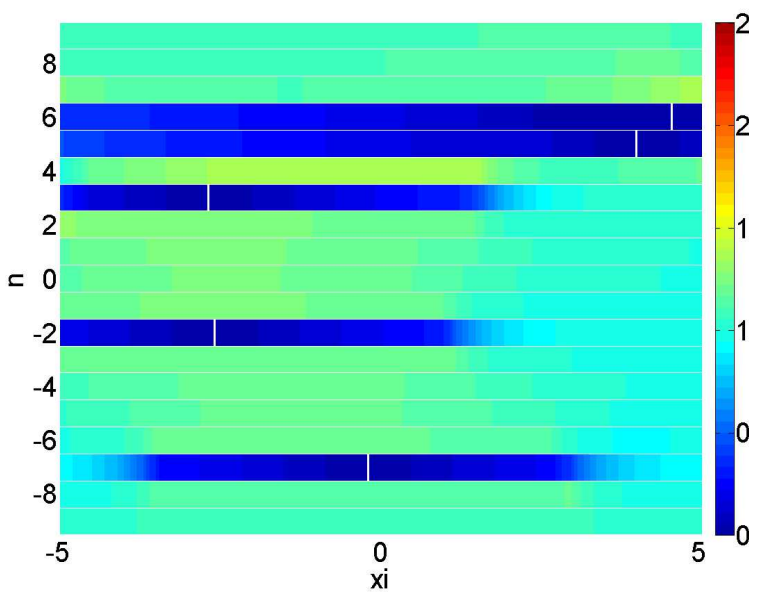

(a) $2050 \mathrm{MPa}-5$ fiber breaks

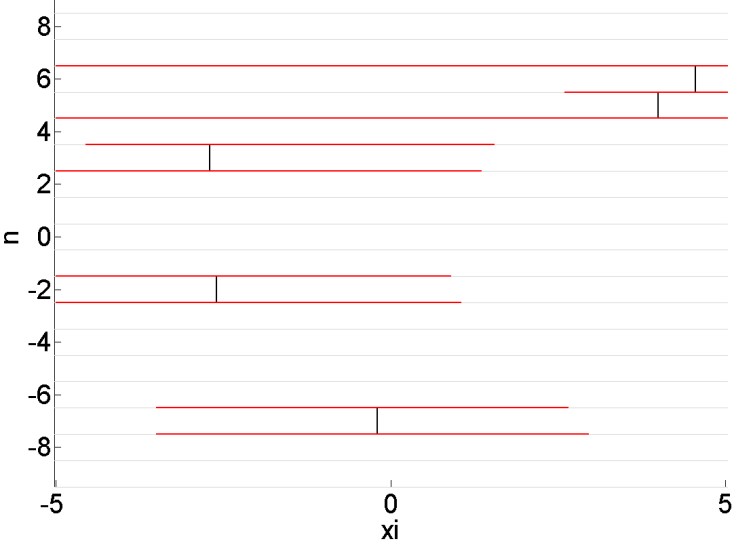

(c) $2050 \mathrm{MPa}$ - 5 fiber breaks and 1191 debonded matrix elements

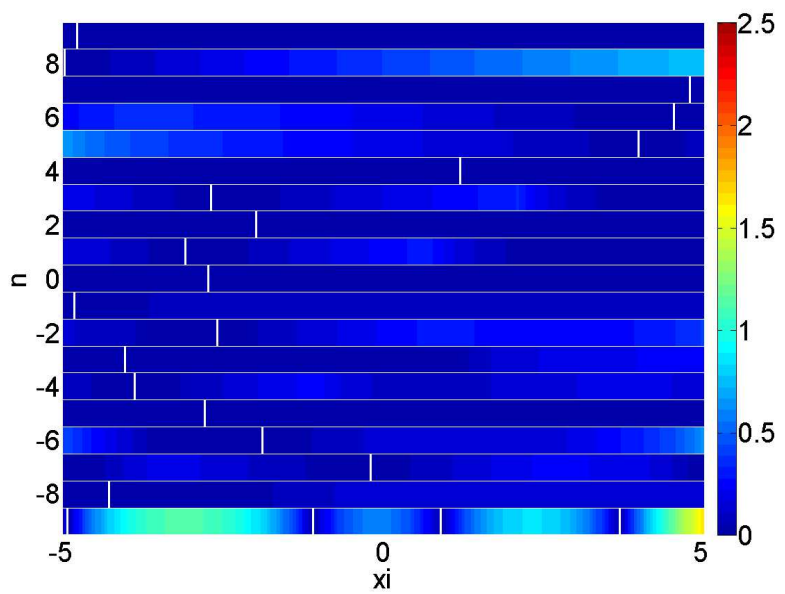

(b) $2100 \mathrm{MPa}-22$ fiber breaks

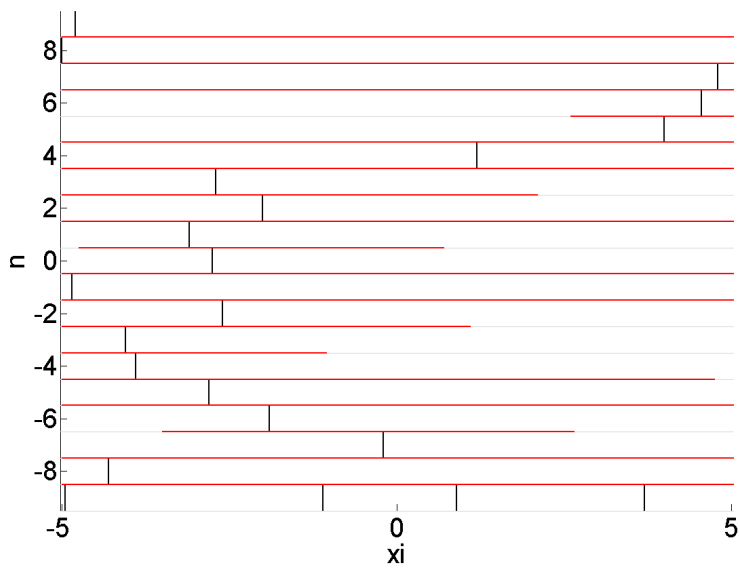

(d) $2100 \mathrm{MPa}$ - 22 fiber breaks and 3242 debonded matrix elements

Figure 11: Damage patterns and overstress factors in the material with debonding

\subsection{Progressive damage in time}

In this section matrix's viscoelastic behavior is introduced and the time-depended damage is observed. First the influence of $G e$, matrix's initial shear stiffness, will be studied, while the other material characteristics remain unchanged (in particular matrix's shear strain yielding limit). Then the influence of an additional shear stress will also be investigated. For these simulations, a permanent far field axial stress is applied to the fibers, it is equal to $1910 M P a$. 


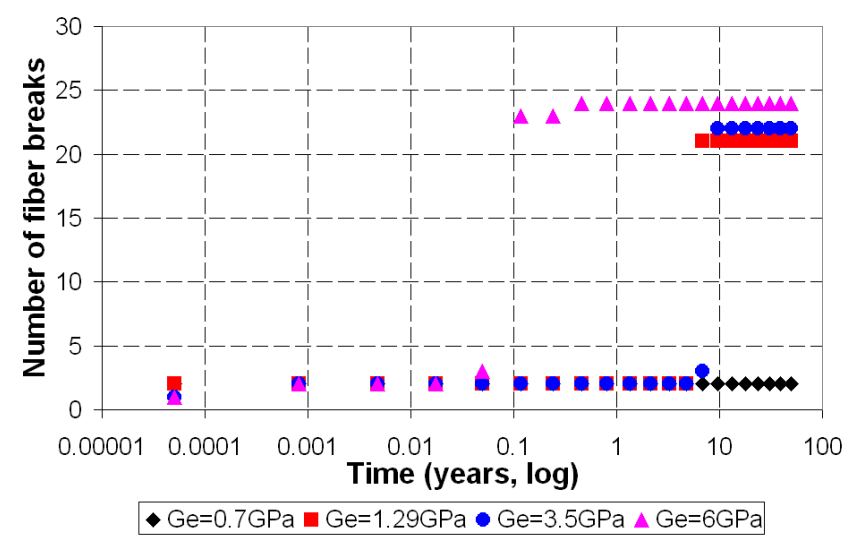

(a) Number of fiber breaks versus time

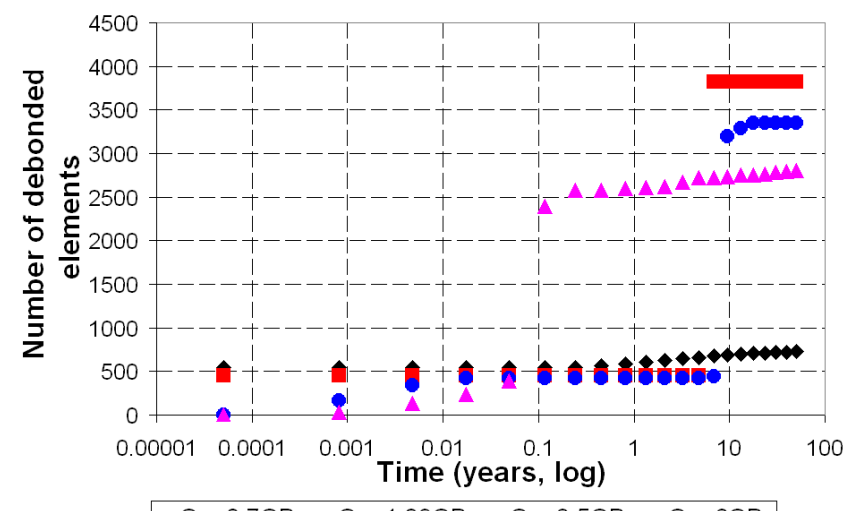

$-\mathrm{Ge}=0.7 \mathrm{GPa} \backsim \mathrm{Ge}=1.29 \mathrm{GPa} \bullet \mathrm{Ge}=3.5 \mathrm{GPa} \triangle \mathrm{Ge}=6 \mathrm{GPa}$

(b) Number of debonded elements versus time

Figure 12: Evolution of damage versus time (logarithmic scale) for samples with various values of the matrix's shear stiffness, subjected to 1910MPa fibers traction stress

\subsubsection{Influence of matrix's shear stiffness}

For these simulations, four values for the matrix's shear stiffness are taken: $G e=0.7 \mathrm{GPa}, 1.29 \mathrm{GPa}$, $3.5 \mathrm{GPa}$ and $6 \mathrm{GPa}$. The influence of this parameter on the long-term durability of the material is studied. The rupture mode, damage evolution and evolution of the apparent modulus are investigated. The sample has the same fibers strengths distribution as described in the figure 8.

The figure 12 gives time evolution of the damage in the material: number of fiber breaks (figure 12 (a)) and number of debonded matrix elements (figure 12 (b)). These figures show a sudden increase in material's damage for samples with $G e \geq 1.29 \mathrm{GPa}$, whereas for the sample with $G e=0.7 \mathrm{GPa}$, the evolution of the damage is smooth and continuous over time. This indicates a modification of the damage process in the material for composites with different matrix's stiffnesses. From approximately 10 years on, the number of fiber breaks for the group of materials with $G e \geq 1.29 \mathrm{GPa}$, is similar ranging from $21(\mathrm{Ge}=1.29 \mathrm{GPa})$ to 24 


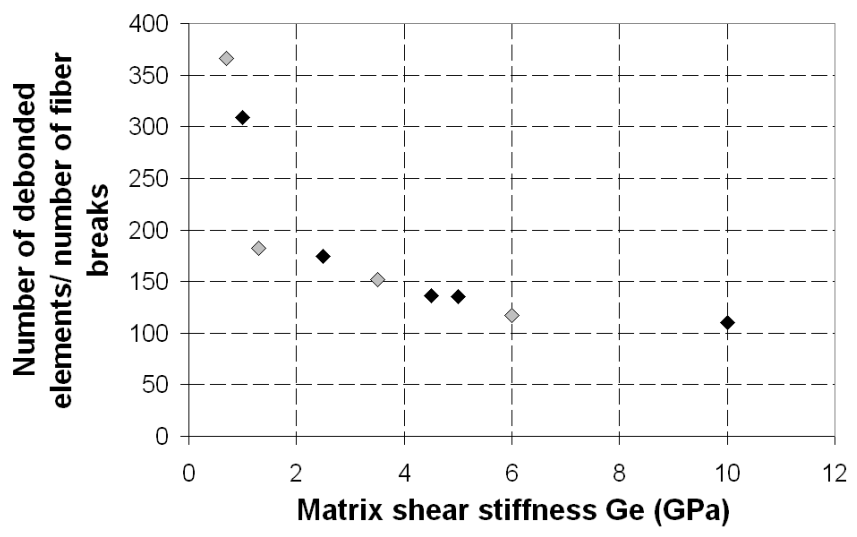

Figure 13: Ratio between debonding and fiber breakage for various matrix stiffnesses

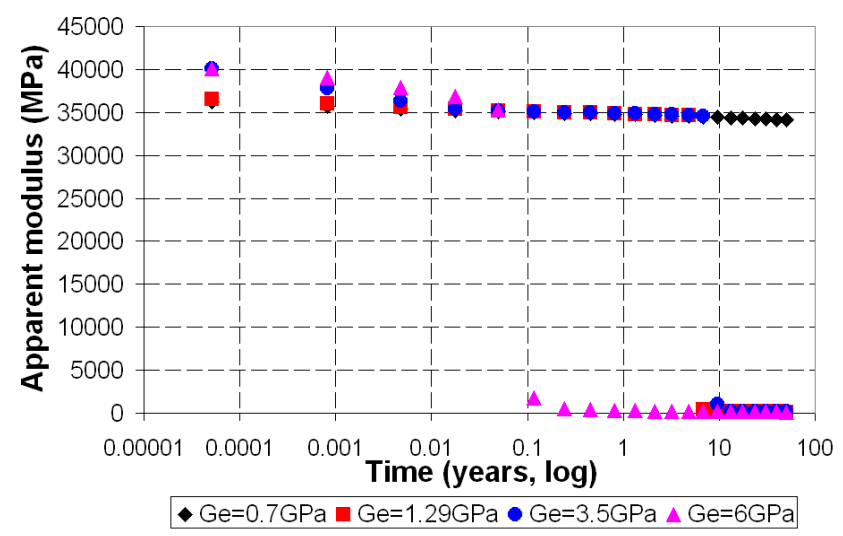

Figure 14: Apparent modulus versus time during the pure traction creep test

$(G e=6 \mathrm{GPa})$, whereas for the material with $G e=0.7 \mathrm{GPa}$, the number of fiber breaks remains constantly equal to 2 . On the other hand, the number of debonded matrix elements for the material with $G e=0.7 \mathrm{GPa}$ is greater than that of the materials with $G e \geq 1.29 \mathrm{GPa}$ before their breakage.

It is also interesting to compare the ratio between the number of debonded matrix elements and the number of fiber breaks at the end of the 50-years creep period for these simulations. The figure 13 summarizes these values. Results from additional simulations with different shear stiffness values were added to the graph in order to determine clear tendencies. Results for $\mathrm{Ge}=0.7,1.29,3.5$ and 6 are highlighted in light grey. What can be seen on this graph is that there is at least two different groups of materials. The first group with $G e<1.29 \mathrm{GPa}$ where damage is predominantly debonding-based (the ratio is higher than 300) and the second group with $G e \geq 1.29 \mathrm{GPa}$, where the ratio between the number of debonded elements and fiber breaks is much lower $(<200)$.

The figure 14 shows the time evolution of the apparent modulus of these samples. In particular a sudden 


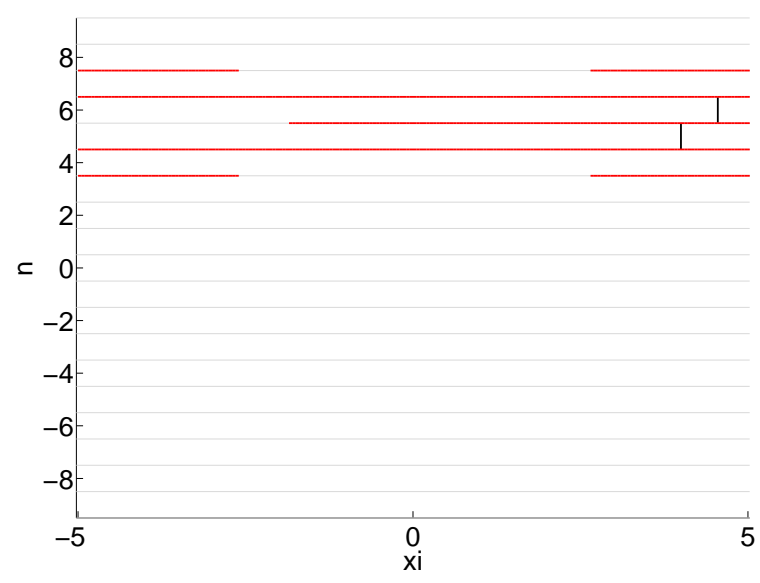

(a) Damage pattern for $G e=0.7 \mathrm{GPa}$, unbroken sample

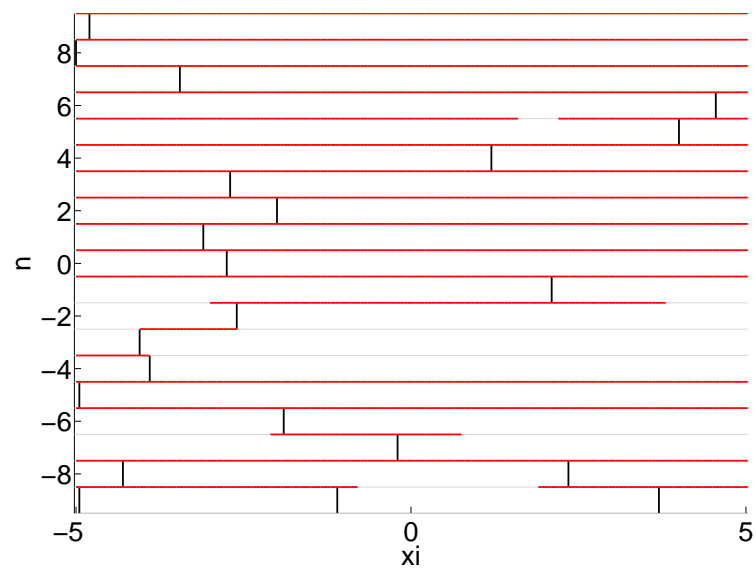

(c) Damage pattern for $G e=3.5 \mathrm{GPa}$, broken sample

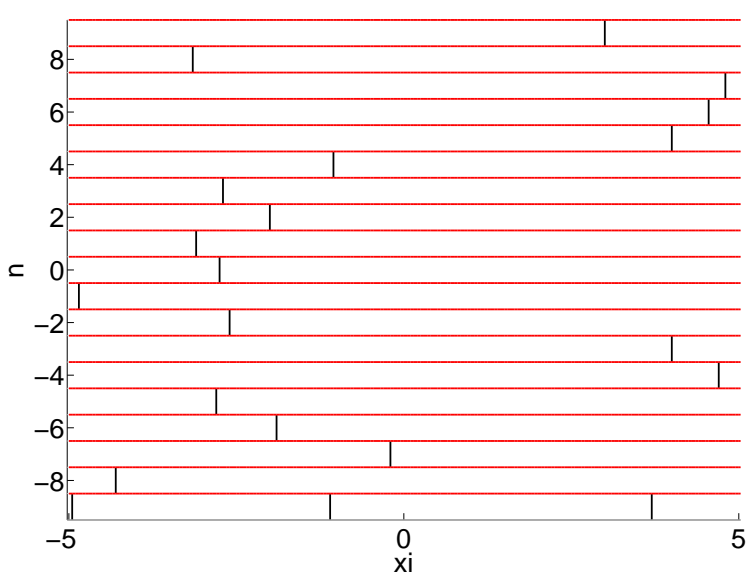

(b) Damage pattern for $G e=1.29 \mathrm{GPa}$, broken sample

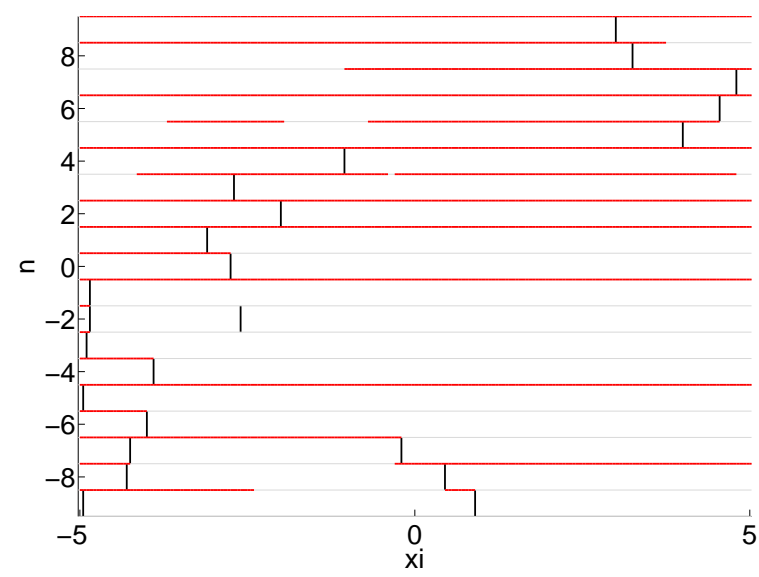

(d) Damage pattern for $G e=6 \mathrm{GPa}$, broken sample

Figure 15: Damage pattern following the breakage of the samples: comparison for different matrix's stiffness values

drop of the apparent modulus for the materials with $G e \geq 1.29 \mathrm{GPa}$ can be noticed. This is due to the fact that a sufficient number of fiber breaks form a macro-cluster completely destroying the material. A macro-cluster in this case is a group of fiber breaks linked by debonded regions, spanning across the whole sample in the transverse direction. For the composite with $G e=0.7 \mathrm{GPa}$, longitudinal splits, isolate broken fibers from the remaining intact fibers, preserving a tractional stiffness of the composite.

Figure 15 gives the damage patterns following the breakage of the samples. This figure confirms the presence of macro-clusters for the materials with $G e \geq 1.29 \mathrm{GPa}$ (figures 15 (b),15 (c), 15 (d)) and longitudinal splits for the material with $G e=0.7 \mathrm{GPa}$ (figure 15 (a)).

These results show that matrix's shear stiffness has an influence on the rupture mode of the material. On 
the examples presented here two groups were identified: rupture based on the development of macro-clusters and rupture mainly initiated through longitudinal splitting. The evolution between these two groups seems to be monotonic in respect to the matrix's stiffness: the group of materials with longitudinal splitting-based rupture has low stiffness values whereas the second group of materials presenting macro-cluster formation has higher stiffness values. Lower stiffness values as well as matrix relaxation lead to the same result on the load sharing in the material: its globalization. Globalization of the load sharing, under certain limits, helps protecting the material: as it was shown in the literature overview, a very stiff and strong interface leads to high overstress factors increasing the number of fiber breaks whereas a weak interface leading to a global load sharing scheme results in a fragmentation of the material and its incapacity to take up broken fibers' load. A medium situation provides the optimal result.

\subsubsection{Influence of an additional shear strain}

In the experimental study presented in the section 2, creep experiments combining bending and torsion were lead. These experimental results motivated a numerical investigation of the influence of an additional shear strain applied to the material in combination with the traction load. Indeed during the experiments when torque is applied to the material, this results in shearing of the material. This shear is either combined with traction or compression resulting from the bending load.

In this section the model is used to simulate time-dependent damage of the sample described in the figure 8. For these simulations two values of the matrix's shear stiffness are taken: $G e=1.29 \mathrm{GPa}$ and $G e=6 \mathrm{GPa}$. A uniform shear strain field equal to $70 \%$ of the limit shear strain is applied to the whole composite. Then a creep test is simulated with an axial far field fiber stress equal to 1910MPa. Comparison between these simulations and simulations where pure traction is applied is performed. Figure 16 gives the damage evolution in four cases: pure traction loading and $G e=1.29 \mathrm{GPa}$, pure traction loading and $G e=6 \mathrm{GPa}$, traction and shear combined and $G e=1.29 \mathrm{GPa}$ and traction and shear combined and $G e=6 \mathrm{GPa}$.

In the case of $G e=6 \mathrm{GPa}$, additional shear strain causes an increase in the lifespan of the material compared to the lifespan under pure traction loading: when pure traction loading is applied, the lifespan (as can be seen in the figure 16 (a)) is approximately 0.05 years, whereas with combined shear it is increased to 1.3 years. At the same time the damage mode is not significantly modified.

In the case of $G e=1.29 \mathrm{GPa}$, the damage process is modified: in pure traction it is fiber breakage based and it becomes predominantly debonding based when shear is applied. The lifespan of the sample is also increased from 4.8 years under pure traction loading to 50 years when traction and shear are combined.

Additional shear in the material leads to additional debonding. This debonding, in the two cases presented here, lead to an increase of the material's lifespan. This is due to the fact that debonded regions isolate the broken fiber, globally, as the relaxation takes place, redistributing its load to the remaining intact fibers. 


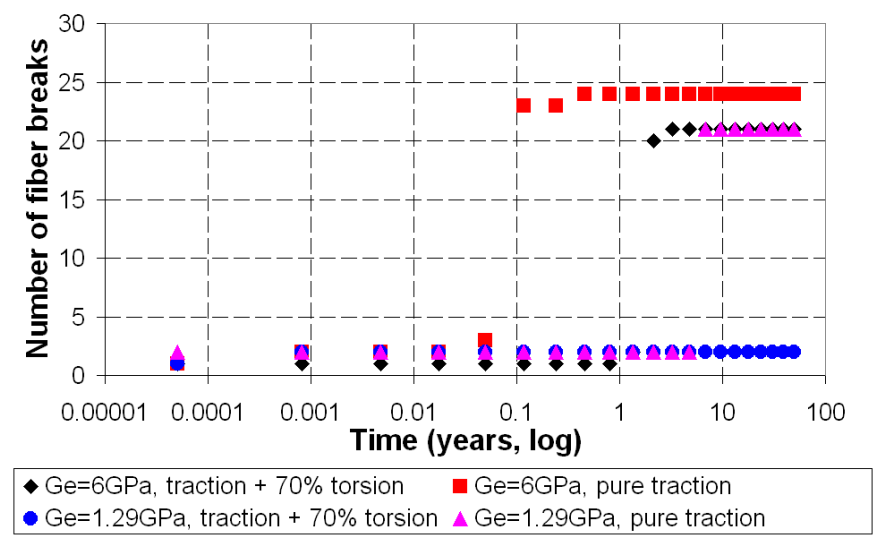

(a) Number of fiber breaks versus time

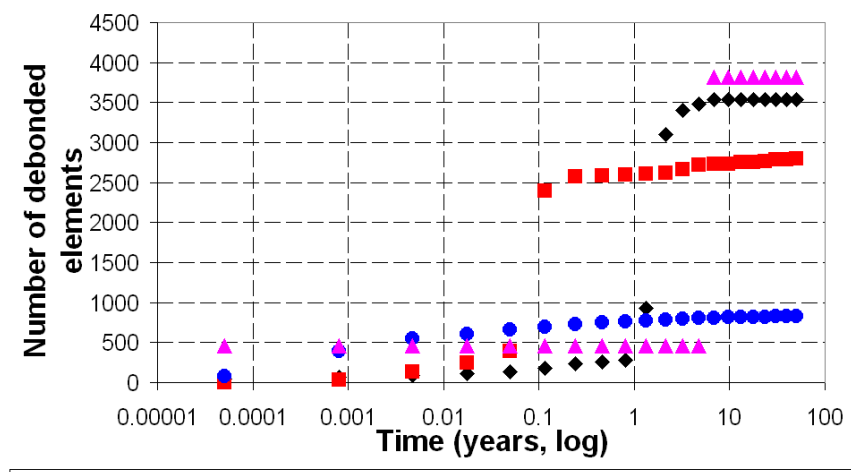

- Ge=6GPa, traction $+70 \%$ torsion $\quad \mathrm{Ge}=6 \mathrm{GPa}$, pure traction

- $\mathrm{Ge}=1.29 \mathrm{GPa}$, traction $+70 \%$ torsion $\Delta \mathrm{Ge}=1.29 \mathrm{GPa}$, pure traction

(b) Number of debonded elements versus time

Figure 16: Evolution of damage versus time (logarithmic scale) for samples with various values of the matrix's shear stiffness, subjected to either 1910MPa fibers traction stress exclusively or combined with $70 \%$ shear strain additional loading

Figure 17 gives the evolution of the apparent modulus during these 4 simulations.

In the figure 18 the corresponding damage patterns are presented. Figures 18 (a) and 18 (b) confirm the modification of the damage mode for $G e=1.29 \mathrm{GPa}$. Figures 18 (c) and 18 (d) show that when shear is applied to the material with $G e=6 \mathrm{GPa}$, fiber breaks become less scattered and a clear fracture appears.

To summarize results of this section, several key facts are to be mentioned: additional shear causes additional debonding, this debonding helps increasing the material's lifespan as it helps isolating broken fibers. The additional shear can modify the rupture mode of the material leading to a fracture initiated by longitudinal splitting. This effect depends on the matrix's shear stiffness: lower stiffness values lead to a modified rupture mode.

In the simulations presented in this section shear loading is combined with traction whereas in the experiments presented in the previous section 2, combined shear and compression also occurred. In this 


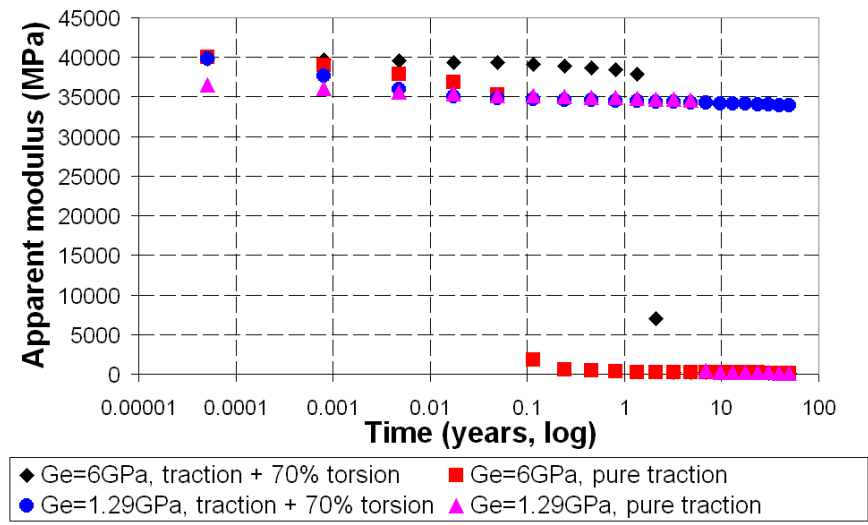

Figure 17: Apparent modulus versus time during the pure traction and traction-shear creep tests

situation, debonding can enhance buckling of the individual fibers leading to the material's premature ruin. This asymmetry in the traction-compression behavior should be introduced in the model in order to demonstrate the reduction of the material's lifespan under flexural loading. 


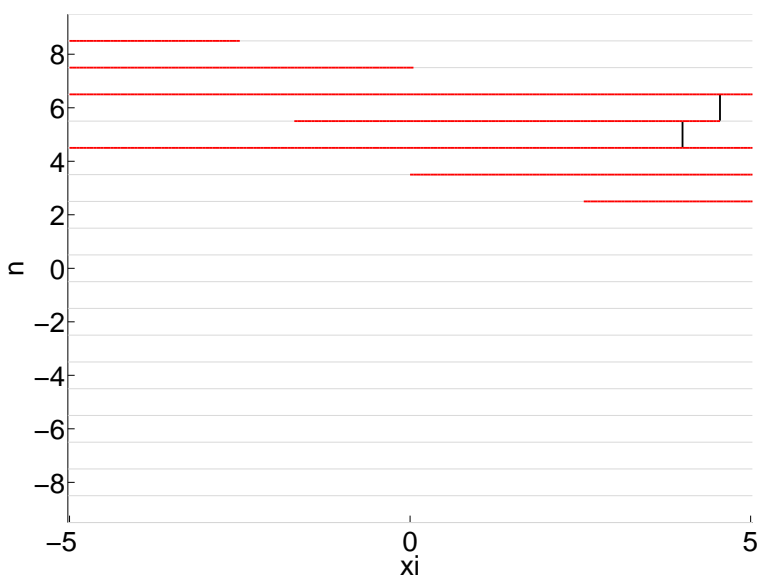

(a) Damage pattern for $G e=1.29 \mathrm{GPa}$ under combined shear and traction loading

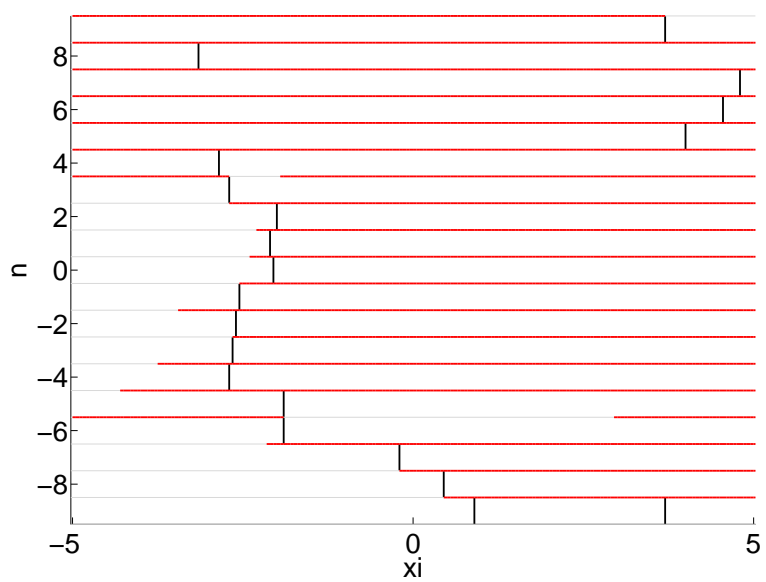

(c) Damage pattern for $G e=6 \mathrm{GPa}$ under combined shear and traction loading

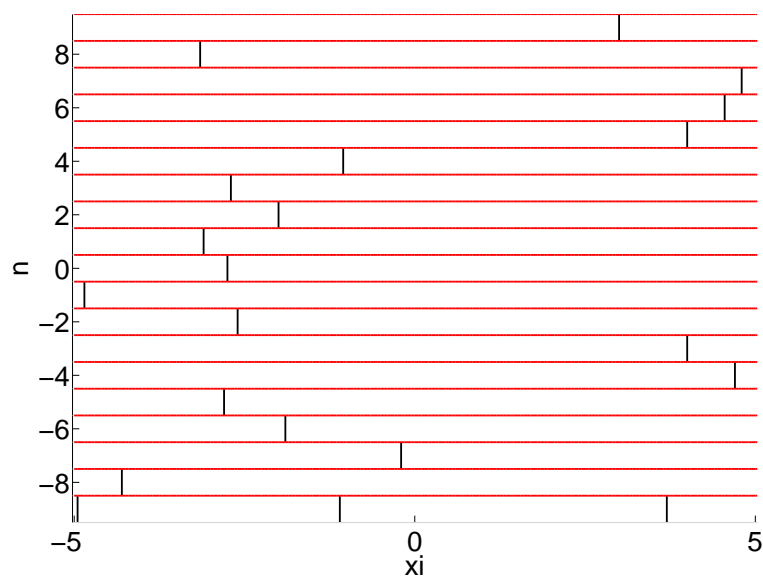

(b) Damage pattern for $G e=1.29 \mathrm{GPa}$ under pure traction loading

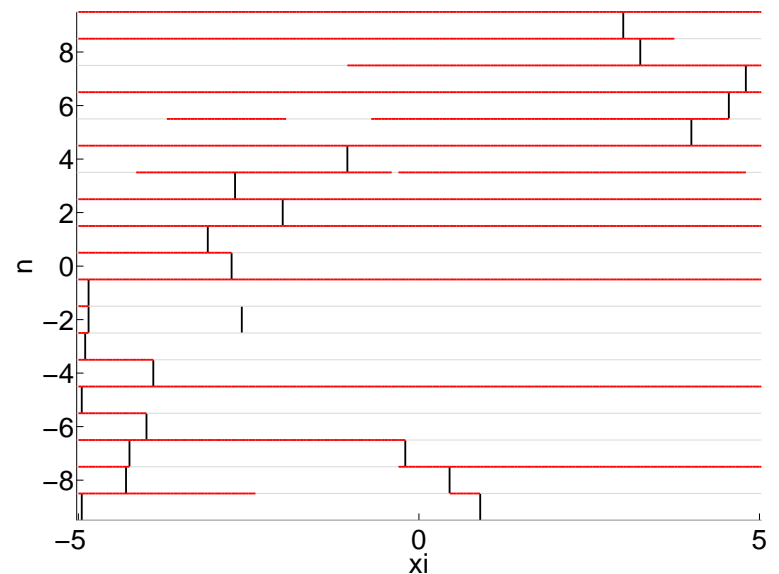

(d) Damage pattern for $G e=6 \mathrm{GPa}$ under pure traction loading

Figure 18: Damage patterns following the breakage of the samples subjected to pure traction and combined traction and shear loadings: comparison for different matrix's stiffness values 


\section{Conclusions and future work}

A shear-lag type model, based on existing previous work of the literature, is further developed in order to simulate progressive debonding occurring in a unidirectional 0o composite material subjected to traction or traction combined with shear. This model includes a stochastic distribution of fiber's strengths, matrix's relaxation in time, progressive debonding at the fiber-matrix interface with a shear strain-based criterion and a residual frictional shear stress also relaxing in time.

First the model is used to investigate the influence of debonding on basic stress transfer phenomena occurring in a composite with an elevated fiber volume fraction. This investigation shows that debonding leads to a globalization of the stress redistribution in the composite: stress is both more equally distributed into the depth of the material and more uniformly distributed on the length of the intact fibers.

This model is then used to simulate progressive damage in a composite material with 19 fibers allowed to break. First the static strength of the sample is assessed by applying increasing tractional loading to the sample. Simulations taking debonding into account and ignoring it were lead. These simulations showed that debonding, as expected, decreased material's static strength. Then creep tests were simulated during a maximum of a 50-years period, where a given tractional load level was applied to the fibers at the far field. In these investigations various mechanical characteristics of the composite's components are used. In particular the influence of matrix's shear stiffness is investigated. Then the influence of an additional shear strain superimposed to the tractional loading is studied. Both lifespan of the sample and type of rupture mode are assessed.

Low stiffness of the matrix encourages debonding (for a given shear strain limit), leading to a modification of the damage process from mainly based on fiber breakage to one predominantly based on the development of longitudinal splitting and debonded zones.

Additional shear seems to have a similar effect on materials with a low matrix shear stiffness: rupture occurs through longitudinal splitting. At the same time the material's lifespan is increased. Indeed debonded regions isolate broken fibers from the intact ones preserving their integrity: as debonding occurs and develops in the relaxing material, localization and maximum overstress factor diminish. Materials with high matrix shear stiffness tend to demonstrate a longer lifespan as well but a more localized fiber rupture pattern when subjected to additional shear.

Further investigation of the influence of various parameters of the composite's configuration are needed. In particular the influence of the fibers volume fraction and parameters of the stochastic distribution of fibers strengths might be of great interest. By the way, a recent article of swolfs and al. [61] confirms in fact the great influence of these parameters. Probabilistic analysis of the composite's lifespan should also be performed using a Monte-Carlo approach. Moreover, new long term experimental work should be led to identify more precisely the required parameters and validate the analysis. Then, Results presented in the 
present paper should allow for a fine-tuning of macro-mechanical models in order to represent in a more precise manner the influence of matrix and interface properties on the creep behavior of composite materials. Finally, the model is a first step toward the modelling of creep rupture of 0o UD composites through combined bending-shear loading which is shown, in the beginning of the present work, as a decisive criterion in real structures. The present model could also become more universally applicable if the axial elastic modulus of the matrix was modelled. This would allow its application to a wider spectrum of composite materials.

\section{Acknowledgements}

Work presented in this article is part of a $\mathrm{PhD}$ thesis financially supported by the French Environment and Energy Management Agency (ADEME) and the French Scientific and Technical Center for Building (CSTB). Experimental samples were provided by Oxylane, manufacturing the Quechua ${ }^{\circledR}$ pop-up tents.

\section{References}

[1] B. Drouin, G. Latour, H. M. Mohamed, More than 10 years successful field applications of FRP bars in Canada, in: Fourth International Conference on Durability and Sustainability of Fibre Reinforced Polymer (FRP) Composites for Construction and Rehabilitation.

[2] E. R. Fyfe, R. Watson, M. McCullagh, More than twenty years of field applications of composites with durability testing and field observations, in: Fourth International Conference on Durability and Sustainability of Fibre Reinforced Polymer (FRP) Composites for Construction and Rehabilitation.

[3] V. Karbhari, J. W. Chin, D. Hunston, B. Benmokrane, T. Juska, R. Morgan, J. Lesko, U. Sorathia, D. Reaynaud, Durability gap analysis for fiber-reinforced polymer composites in civil infrastructure, Journal of Composites for Construction 7 (2003) 238-247.

[4] Y. Shao, J. Shanmugam, Deflection creep of pultruded composite sheet piling, Journal of Composites for Construction (ASCE) (2004) 471-479.

[5] Y. Shao, J. Shanmugam, Moment capacities and deflection limits of PFRP sheet piles, Journal of Composites for Construction (ASCE) (2006) 520-528.

[6] Y. Shao, Characterization of a pultruded FRP sheet pile for waterfront retaining structures, Journal of material in Civil Engineering 18 (2006) 626-633.

[7] Z. Wu, A. Mirmiran, Z. Zhu, J. Swanson, Flexural behavior of prestressed FRP tubular bridge deck, Composites: Part B 40 (2009) 125-133.

[8] J. Hillman, Sustainable structures using hybrid-composite beams, in: Fourth International Conference on Durability and Sustainability of Fibre Reinforced Polymer (FRP) Composites For Construction and Rehabilitation.

[9] C. Douthe, J. Caron, O. Baverel, Gridshell structures in glass fibre reinforced polymers, Construction and Building Materials 24 (2010) 1580-1589.

[10] C. Douthe, O. Baverel, J. Caron, Form-finding of a grid shell in composite materials, Journal of the International Association for Shell and Spatial Structures 47 (2006) 53-62.

[11] N. Kotelnikova-Weiler, Optimisation mécanique et énergétique d'enveloppes en matériaux composites pour les bâtiments, Ph.D. thesis, Université Paris-Est, 2012.

[12] F. Ceroni, E. Cosenza, M. Gaetano, M.Pecce, Durability issues of FRP rebars in reinforced concrete members, Cement \& Concrete Composites 28 (2006) 857-868. 
[13] L. Bank, A. Mosallam, Creep and failure of a full-size fiber-reinforced plastic pultruded frame, Composites Engineering 2 (1992) 213-227.

[14] H. Kim, Y. Park, Y. You, C. Moon, Short-term durability test for GFRP rods under various environmental conditions, Composite Structures 83 (2008) 37-47.

[15] C. Zhou, L. Schafler, I. Beyerlein, Stress concentrations in graphite/epoxy model composites during creep at room temperature and elevated temperatures, Journal of Composite Materials 38 (2004) 417-433.

[16] A. Scott, M. Mavrogordato, P. Wright, I. Sinclair, S. Spearing, In situ fibre fracture measurement in carbon-epoxy laminates using high resolution computed tomography, Composites Science and Technology 71 (2011) 1471-1477.

[17] G. Foray, M. R'Mili, A. Descamps-Mandine, Comparative fiber defect size distribution analysis by AFM and bundle tensile test, in: Journées Nationales sur les Composites 17.

[18] J. Gordon, Structures et Matï¡œriaux. L'explication mï¿œcanique des formes., Belin, 1994.

[19] I. Beyerlein, C. Zhou, L. Schadler, A time-dependent micro-mechanical fiber composite model for inelastic zone growth in viscoelastic matrices, Internationa Journal of Solids and Structures 40 (2003) 2171-2194.

[20] F. Zhao, N. Takeda, Effect of interfacial adhesion and statistical fiber strength on tensile strength of unidirectional glass fiber/epoxy composites. Part 1: experiment results, Composites: Part A 31 (2000) 1203-1214.

[21] C. Zhou, L. Schadler, I. Beyerlein, Time-dependent micromechanical behavior in graphite/epoxy composites under constant load: a combined experimental and theretical study, Acta Materialia 50 (2002) 365-377.

[22] A. Diaz Diaz, J. Caron, Interface plasticity and delamination onset prediction, Mechanics of Materials 38 (2006) 648-663.

[23] L. Mishnaevsky, P. Brøndsted, Micromechanical modeling of damage and fracture of unidirectional fiber reinforced composites: A review, Computational Materials Science 44 (2009) 1351-1359.

[24] H. Cox, The elasticity and strength of paper and other fibrous materials, British Journal of Applied Physics 3 (1952) $72-79$.

[25] J. Hedgepeth, Stress Concentrations in Filamentary Structures, Technical Report, National Aeronautics and Space Administration, 1961.

[26] B. Rosen, Tensile failure of fibrous composites, AIAA Journal 2 (1964) 1985-1991.

[27] C. Zweben, Tensile failure of fiber composites, AIAA Journal 6 (1968) 2325-2331.

[28] P. Van Dyke, J. M. Hedgepeth, Stress concentrations from single-filament failures in composite materials, Textile Research Journal 39 (1969) 618-626.

[29] A. Sastry, S. Phoenix, Load redistribution near non-aligned fibre breaks in a two-dimensional unidirectional composite using break influence supersposition, Journal of Materials Science Letters 12 (1993) 1596-1599.

[30] J. A. Nairn, On the use of shear-lag methods for analysis of stress transfer in unidirectional composites, Mechanics of Materials 26 (1997) 63-80.

[31] I. Beyerlein, S. Phoenix, R. Raj, Time evolution of stress redistribution around multiple fiber breaks in a composite with viscous and viscoelastic matrices, Internationa Journal of Solids and Structures 35 (1998) 3177-3211.

[32] C. Landis, M. McGlockton, R. McMeeking, An improved shear-lag model for broken fibers in composite materials, Journal of Composite Materials 33 (1999) 667-680.

[33] C. M. Landis, R. McMeeking, Stress concentrations in composites with interface sliding, matrix stiffness and uneven fiber spacing using shear-lag theory, Internationa Journal of Solids and Structures 36 (1999) 4333-4361.

[34] C. Landis, I. Beyerlein, R. McMeeking, Micromechanical simulation of the failure of fiber reinforced composites, Journal of Mechanics and Physics of Solids 48 (2000) 621-648.

[35] B. J. Thuruthimattam, A. M. Waas, A. S. Wineman, Stress transfer modeling in viscoelastic polymer matrix composites, International Journal of Non-Linear Mechanics 36 (2001) 69-87.

[36] T. Okabe, N.Takeda, Y. Kamoshida, M. Shimizu, W. Curtin, A 3D shear-lag model considering micro-damage and 
statistical strength prediction of unidirectional fiber-reinforced composites, Composite Science and Technology 61 (2001) $1773-1787$.

[37] S. Mahesh, J. Hanan, E. Ütündag, I. Beyerlein, Shear-lag model for a single fiber metal matrix composite with elasto-plastic matrix and a slipping interface, Internationa Journal of Solids and Structures 41 (2004) 4197-4218.

[38] J. Koyanagi, H. Hatta, F. Ogawa, H.Kawada, Time-dependent reduction of tensile strength caused by interfacial degradation under constant strain duration in UD-CFRP, Journal of Composite Materials 41 (2007) 3007-3026.

[39] X. Chen, I. Beyerlein, L. C. Brinson, Curved-fiber pull-out model for nanocomposites. Part 1: Bonded stage formulation, Mechanics of Materials 41 (2009) 279-292.

[40] Z. Xia, W. Curtin, P. Peters, Multiscale modeling of failure in metal matrix composites, Acta Materialia 49 (2001) 273-287.

[41] S. Blassiau, A. Thionnet, A. Bunsell, Micromechanisms of load transfer in a unidirectional carbon fibre-reinforced epoxy composite due to fibre failures: Part 1: Micromechanisms and 3d analysis of load transfer: the elastic case, Composite Structures 74 (2006) 303-318.

[42] A. Scott, I. Sinclair, S. Spearing, A. Thionnet, A. Bunsell, Damage accumulation in a carbon/epoxy composite: Comparison between a multiscale model and computed tomography experimental results, Composites: Part A 43 (2012) $1514-1522$.

[43] F. Wang, X. Li, J. Zhang, L. Li, L. M. Keer, Micromechanical modelling of the progressive failure in unidirectional composites reinforced with bamboo fibres, Mechanics of Materials 94 (2016) 180-192.

[44] D. Lagoudas, C.-Y. Hui, S. Phoenix, Time evolution of overstress profiles near broken fibers in a composite with a viscoelastic matrix, Internationa Journal of Solids and Structures 25 (1989) 45-66.

[45] S. Blassiau, A. Thionnet, A. Bunsell, Micromechanisms of load transfer in a unidirectional carbon fibre-reinforced epoxy composite due to fibre failures: Part 2: Influence of viscoelastic and plastic matrices on the mechanisms of load transfer, Composite Structures 74 (2006) 319-331.

[46] V. Monfared, F. Mondali, A displacement based model to determine the steady state creep strain rate of short fiber composites, Composites Science and Technology 107 (2015) 18-28.

[47] R. M. Guedes, J. Morais, A. T. Marques, A. H. Cardon, Prediction of long-term behaviour of composite materials, Computers and Structures 76 (2000) 183-194.

[48] S. Mahesh, S. Phoenix, Lifetime distributions for unidirectional fibrous composites under creep-rupture loading., International Journal of Fracture 127 (2004) 303-360.

[49] S. Blassiau, A. Thionnet, A. Bunsell, Micromechanisms of load transfer in a unidirectional carbon fibre-reinforced epoxy composite due to fibre failures: Part 3. Multiscale reconstruction of composite behaviour, Composite Structures 83 (2008) $312-323$.

[50] B. Nedjar, A time dependent model for unidirectional fibre-reinforced composites with viscoelastic matrices, Internationa Journal of Solids and Structures 48 (2011) 2333-2339.

[51] B. Nedjar, Directional damage gradient modeling of fiber/matrix debonding in viscoelastic UD composites, Composite Structures 153 (2016) 895-901.

[52] W. Na, G. Lee, M. Sung, H. N. Han, W.-R. Yu, Prediction of the tensile strength of unidirectional carbon fiber composites considering the interfacial shear strength, Composite Structures (2017).

[53] J. Koyanagi, Comparison of a viscoelastic frictional interface theory with a kinetic crack growth theory in unidirectional composites, Composites Science and Technology 69 (2009) 2158-2162.

[54] J. Koyanagi, S. Yoneyama, A. Nemoto, J. Melo, Time and temperature dependence of carbon/epoxy interface strength, Composites Science and Technology 2010 (70) 1395-1400.

[55] J. Koyanagi, S. Yoneyama, K. Eri, P. D. Shah, Time dependency of carbon/epoxy interface strength, Composite Structures 
92 (2010) 150-154.

[56] J. Koyanagi, A. Yoshimura, H. Kawada, Y. Aoki, A numerical simulation of time-dependent interface failure under shear and compressive loads in single-fiber composites, Applied Composite Materials 17 (2010) 31-41.

[57] I. J. Beyerlein, S. L. Phoenix, Stress concentrations around multiple fiber breaks in an elastic matrix with local yielding or debonding using quadratic influence superposition, Journal of the Mechanics and Physics of Solids 44 (1996) 1997-2039.

[58] I. Beyerlein, S. Phoenix, A. Sastry, Stress concentrations around aligned fiber breaks in a unidirectional composite with an elastic-plastic matrix, in: Simulation of Materials Processing: Theory, Methods and Applications.

[59] S. Blassiau, A. Thionnet, A. Bunsell, Three-dimensional analysis of load transfer micro-mechanisms in fibre/matrix composites, Composites Science and Technology 69 (2009) 33-39.

[60] P. Zinck, Sample size dependence of flaw distributions for the prediction of brittle solids strength using additive Weibull bimodal distributions, Engineering Fracture Mechanics 78 (2011) 1323-1327.

[61] Y. Swolfs, R. M. McMeeking, I. Verpoest, L. Gorbatikh, Matrix cracks around fibre breaks and their effect on stress redistribution and failure development in unidirectional composites, Composites Science and Technology 108 (2015) $16-22$. 